\title{
On a Class of \\ Parabolic Integro-Differential Equations
}

\author{
W. Kohl
}

Abstract. Existence and uniqueness results for the integro-differential equation

$$
u_{1}(x, t)-a u_{x x}(x, t)=c(x, t) u(x, t)+\int_{0}^{1} k(s, x) h(s, t, u(s, t)) d s+f(x, t) \quad((x, t) \in Q)
$$

subject to the boundary condition

$$
u(x, t)=\varphi(x, t) \quad((x, t) \in R)
$$

and, especially, for the linear case $h(s, t, u)=u$ are given. To this end, this equation is written as operator equation in a suitable Hölder space. The main tools are the calculation of the spectral radius in the linear case, and fixed point principles in the nonlinear case.

Keywords: Integro-differential equations, parabolic operators, multiplication operators, integral operators, Hölder spaces, heat potential, existence and uniqueness of solutions, Neumann series, fixed point principle

AMS subject classification: 47 G 20, 47 H 10, 47 H 30, 45 K 05, 35 K 99, 26 B 35

\section{Introduction}

In this paper we study existence and uniqueness results for the parabolic integrodifferential equation

$$
u_{t}(x, t)-a u_{x x}(x, t)=c(x, t) u(x, t)+\int_{0}^{1} k(s, x) u(s, t) d s+f(x, t) \quad((x, t) \in Q)
$$

subject to the boundary condition

$$
u(x, t)=\varphi(x, t) \quad((x, t) \in R)
$$

Here $c: Q \rightarrow \mathbb{R}, k:(0,1) \times(0,1) \rightarrow \mathbb{R}, f: Q \rightarrow \mathbb{R}$, and $\varphi: R \rightarrow \mathbb{R}$ are given functions, where $Q=(0,1) \times(0, T)$ and $R=\bar{Q} \backslash Q$ is its parabolic boundary; the parameter $a$ is a real constant. Equations of this type occur in the mathematical modelling of

W. Kohl: Neue Vockenroter Steige 17, D-97877 Wertheim

ISSN 0232-2064 / S 2.50 (C) Heldermann Verlag Berlin 
various transport problems, e.g., describing the propagation of radiation through the atmospheres of planets and stars $[4,5]$, or the transfer of neutrons through thin plates and membranes in nuclear reactors [6]. In the case $a=0$ this boundary value problem has been studied in the recent survey paper [1]. By means of a simple scaling argument we may suppose that $a=1$.

If we introduce the differential operator

$$
L u(x, t)=u_{t}(x, t)-u_{x x}(x, t)
$$

the multiplication operator

$$
C u(x, t)=c(x, t) u(x, t)
$$

and the partial integral operator

$$
K u(x, t)=\int_{0}^{1} k(s, x) u(s, t) d s,
$$

we may write (1) as operator equation

$$
L u=(C+K) u+f .
$$

Our strategy for proving existence (and sometimes also uniqueness) of solutions to the operator equation (6) with boundary condition (2) is standard: First we give conditions under which the classical parabolic boundary value problem

$$
\left.\begin{array}{rr}
L u=f & \text { in } Q \\
u=\varphi & \text { on } R
\end{array}\right\}
$$

has a unique solution for each $f$ and $\varphi$ in some suitable Banach space; this allows us to define the operator $L^{-1}$ on this Banach space. Afterwards we pass from the operator equation (6) to the equivalent equation

$$
u-L^{-1}(C+K) u=L^{-1} f
$$

and try to find conditions under which the spectral radius of the operator $L^{-1}(C+K)$ is less than 1 , in order to apply the classical Neumann series. In fact it turns out that the spectral radius of the linear operator $L^{-1}(C+K)$ is 0 , if we take a Hölder space as underlying Banach space of the operator equation (8).

Apart from the linear equation (1), we will also be interested in the nonlinear equation

$$
\begin{aligned}
& u_{t}(x, t)-a u_{x x}(x, t) \\
& \quad=c(x, t) u(x, t)+\int_{0}^{1} k(s, x) h(s, t, u(s, t)) d s+f(x, t) \quad((x, t) \in Q)
\end{aligned}
$$


where $h: Q \times \mathbb{R} \rightarrow \mathbb{R}$ is some Carathéodory function. Introducing the nonlinear Nemytskij operator

$$
H u(x, t)=h(x, t, u(x, t))
$$

generated by the function $h$, we may write (9) again as operator equation

$$
L u=(C+K H) u+f
$$

If we suppose again that the parabolic operator $L$ be invertible in some Banach space, we end up, analogously to (8), with the nonlinear operator equation

$$
u-L^{-1}(C+K H) u=L^{-1} f
$$

which may be studied by several (classical and non-classical) fixed point principles.

The plan of this paper is as follows. First we introduce some special spaces of continuous functions in which the operator (3) and its inverse have particularly "nice" properties. In Lemma 1 and Lemma 2 we describe some features of the inverse operator by estimations which are not only useful for later functional analytic considerations. These estimations fill also a gap in the literature of the heat equation. So we aimed at thoroughness in proving them. Afterwards we give sufficient conditions under which the operators (4) and (5) are bounded in these spaces. It turns out that analogous results for the nonlinear operator (10) are much more involved. Finally, we show how our results give existence and uniqueness results for solutions of the linear boundary value problem (1)/(2) and the nonlinear boundary value problem (9)/(2).

\section{The heat potential}

Following the theory of the heat equation in the book of J. R. Cannon [2: Chapter 19] we know that the inhomogeneous heat equation (7) is invertible, if the data $f$ is bounded and uniformly Hölder continuous on each compact subset of the domain under consideration. A detailed discussion of the inverse operator $L^{-1}$ in the case of the infinite set $(-\infty,+\infty) \times(0, T]$ is given in this book. Because we could not find similiar investigations for the finite set $Q$ in the literature, we turn now our attention to this case. The inverse $L^{-1}$ corresponding to the rectangular set $Q$ can be represented as a linear Volterra operator

$$
L^{-1} f(i, t)=\int_{0}^{t} \int_{0}^{1} \Gamma(x, t ; \xi, \tau) f(\xi, \tau) d \xi d \tau
$$

which is generated by the Green's function $\Gamma$ for the Dirichlet problem [3: p. 195]. This function can be expressed with the help of the $\theta$-function

$$
\theta(x, t ; \xi, \tau)=\sum_{n=-\infty}^{+\infty} \exp \frac{-n^{2}+n(x-\xi)}{t-\tau}-\sum_{n=-\infty}^{+\infty} \exp \frac{-n^{2}+n(x+\xi)-x \xi}{t-\tau}
$$


and the heat kernel

in the form

$$
\gamma(x, t)=\frac{e^{-x^{2} / 4 t}}{\sqrt{4 \pi t}}
$$

$$
\Gamma(x, t ; \xi, \tau)= \begin{cases}\gamma(x-\xi, t-\tau) \theta(x, t ; \xi, \tau) & \text { if } x, \xi \in \mathbb{R} \text { and } \tau<t \\ 0 & \text { if } x, \xi \in \mathbb{R} \text { and } \tau \geq t\end{cases}
$$

Thus the function $\Gamma$ is infinitely often continuously differentiable for all $x, \xi \in \mathbb{R}$ and $\tau<t$. For fixed $(\xi, \tau) \in \mathbb{R}^{2}$ it solves the heat equation for all $x \in \mathbb{R}$ and $t>\tau$, while for fixed $(x, t) \in \mathbb{R}^{2}$ it is a solution of the adjoint heat equation for all $\xi \in \mathbb{R}$ and $\tau<t$. Moreover, we have the boundary properties

$$
\begin{aligned}
\Gamma(0, t ; \xi, \tau)=\Gamma(1, t ; \xi, \tau) & =0 \quad(\xi \in \mathbb{R}, \tau<t) \\
\Gamma_{x x}(0, t ; \xi, \tau)=\Gamma_{x x}(1, t ; \xi, \tau) & =0 \quad(\xi \in \mathbb{R}, \tau<t) .
\end{aligned}
$$

In order to investigate the operator $\dot{L}^{-1}$ we introduce for fixed $\varepsilon>0$ the family of functions $u_{h}$ with

$$
u_{h}(x, t)=\int_{0}^{t-h} \int_{0}^{1} \Gamma(x, t ; \xi, \tau) f(\xi, \tau) d \xi d \tau \quad\left((x, t) \in \mathbb{R} \times[\varepsilon, T], 0<h<\frac{\varepsilon}{2}\right) .
$$

Now the singularity $(x, t)$ of the Green's function lies not in the domain of integration. So we conclude assuming $f \in L^{\infty}(Q)$ that each function $u_{h}$ is infinitely often continuously differentiable with respect to $x$ and differentiating under the integral sign is permitted

$$
\frac{\partial^{k} u_{h}}{\partial x^{k}}(x, t)=\int_{0}^{t-h} \int_{0}^{1} \frac{\partial^{k} \Gamma}{\partial x^{k}}(x, t ; \xi, \tau) f(\xi, \tau) d \xi d \tau \quad(k \in \mathbb{N}) .
$$

In the case $f \in C^{0}(\bar{Q})$ we may differentiate (17) with respect to $t$ to yield identity (18) for all $(x, t) \in \mathbb{R} \times[\varepsilon, T], 0<h<\frac{\varepsilon}{2}$

$$
\frac{\partial u_{h}}{\partial t}(x, t)=\frac{\partial^{2} u_{h}}{\partial x^{2}}(x, t)+\int_{0}^{1} \Gamma(x, t ; \xi, t-h) f(\xi, t-h) d \xi .
$$

The properties of functions $L^{-1} f$ with $f \in L^{\infty}(Q)$ are summarized in the following lemma. Let $C^{\alpha, 0}(\bar{Q})$ denote, as usual, the set of all $v \in C^{0}(\bar{Q})$ such that there exists a $c>0$ with

$$
h \ddot{o l} l_{\alpha}(v(\cdot, t)):=\sup _{x, y \in[0,1], x \neq y} \frac{|v(x, t)-v(y, t)|}{|x-y|^{\alpha}} \leq c \quad(t \in[0, T]) .
$$

Lemma 1. For $f \in L^{\infty}(Q)$, the heat potential

$$
u(x, t)=L^{-1} f(x, t)=\int_{0}^{t} \int_{0}^{1} \Gamma(x, t ; \xi, \tau) f(\xi, \tau) d \xi d \tau
$$


has the following properties:

(a) $u \mid R=0$ and $u \in C^{0}(\bar{Q})$ with $\sup _{x \in[0,1]}|u(x, t)| \leq c_{1}(T)\|f\|_{\infty} t$ where $\|f\|_{\infty}=$ $\inf _{\mu(N)=0} \sup _{(x, t) \in Q \backslash N}|f(x, t)|$ is a norm in $L^{\infty}(Q)$.

(b) $u_{x} \in C^{0}(\bar{Q})$ with $u_{x}(x, t)=\int_{0}^{t} \int_{0}^{1} \Gamma_{x}(x, t ; \xi, \tau) f(\xi, \tau) d \xi d \tau, u_{x}(x, 0) \equiv 0$, and $\sup _{x \in[0,1]}\left|u_{x}(x, t)\right| \leq c_{2}(T)\|f\|_{\infty} \sqrt{t}$. $[0, T])$.

(c) $u_{x} \in C^{\frac{1}{3}, 0}(\bar{Q})$, i.e. $\left|u_{x}(x+\delta, t)-u_{x}(x, t)\right| \leq c_{3}(T)\|f\|_{\infty}|\delta|^{\frac{1}{3}}(x+\delta, x \in[0,1], t \in$

Proof. Part (a): Since the $\theta$-function is bounded on the set

$$
D=\left\{(x, t ; \xi, \tau) \in \mathbb{R}^{4} \mid x, \xi \in[0,1], t, \tau \in[0, T], \tau<t\right\},
$$

we may estimate the function $u$ by

$$
\begin{aligned}
|u(x, t)| & \leq \int_{0}^{t} \int_{0}^{1}|\Gamma(x, t ; \xi, \tau)||f(\xi, \tau)| d \xi d \tau \\
& \leq \underbrace{\sup _{D}|\theta(x, t ; \xi, \tau)|}_{=c_{1}(T)}\|f\|_{\infty} \int_{0}^{t} \int_{-\infty}^{\infty} \gamma(x-\xi, t-\tau) d \xi d \tau \\
& \leq c_{1}(T)\|f\|_{\infty} t
\end{aligned}
$$

So the function $u$ is well defined on $\bar{Q}$ and satisfies the asserted inequality. Furthermore, we estimate the difference $u-u_{h}$ by

$$
\begin{aligned}
\left|u(x, t)-u_{h}(x, t)\right| & \leq \int_{t-h}^{t} \int_{0}^{1}|\Gamma(x, t ; \xi, \tau)||f(\xi, \tau)| d \xi d \tau \\
& =c_{1}(T)\|f\|_{\infty} h .
\end{aligned}
$$

Taking a sequence $\left(h_{n}\right)$ with $\lim _{n \rightarrow \infty} h_{n}=0$ the sequence $\left(u_{h_{n}}\right)$ of continuous functions converges uniformly on $[0,1] \times[\varepsilon, T]$ towards the function $u$ for all $\varepsilon>0$. Hence we have $u \in C^{0}([0,1] \times(0, T])$ and the function $u(\cdot, t)$ possesses zero boundary values

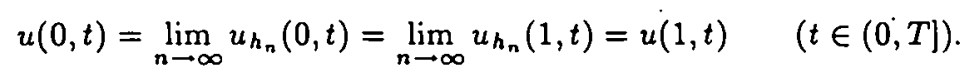

Moreover, the estimation $|u(x, t)| \leq c_{1}(T)\|f\|_{\infty} t$ shows that $u(x, t) \rightarrow 0$ as $t \searrow 0$ uniformly for all $x \in[0,1]$, and we conclude $u \in C^{0}(\bar{Q})$ with $u \mid R=0$.

Part (b): The existence of the first derivative $u_{x}$ of the heat potential is based on the crucial inequality

$$
\int_{0}^{1}\left|\Gamma_{x}(x, t ; \xi, \tau)\right| d \xi \leq c(T) \frac{1}{\sqrt{t-\tau}}
$$


which we prove first. The product rule and further estimation leads to

$$
\begin{aligned}
& \int_{0}^{1}\left|\Gamma_{x}(x, t ; \xi, \tau)\right| d \xi \\
& \quad \leq \int_{0}^{1}\left|\theta_{x}(x, t ; \xi, \tau)\right| \gamma(x-\xi, t-\tau) d \xi+\int_{0}^{1}|\theta(x, t ; \xi, \tau)|\left|\gamma_{x}(x-\xi, t-\tau)\right| d \xi \\
& \quad=: J_{1}+J_{2} .
\end{aligned}
$$

We estimate the integral $J_{1}$ by the two integrals

$$
\int_{0}^{1}\left|\theta_{x}(x, t ; \xi, \tau)\right| \gamma(x-\xi, t-\tau) d \xi \leq A+B
$$

with

$$
A=\int_{0}^{1} \sum_{n=-\infty}^{n=+\infty} \frac{|n|}{t-\tau} \exp \left(-\frac{n^{2}}{t-\tau}+n \frac{x-\xi}{t-\tau}\right) \gamma(x-\xi, t-\tau) d \xi
$$

and

$$
B=\int_{0}^{1} \sum_{n=-\infty}^{n=+\infty} \frac{|n-\xi|}{t-\tau} \exp \left(-\frac{n^{2}}{t-\tau}+n \frac{x+\xi}{t-\tau}-\frac{x \xi}{t-\tau}\right) \gamma(x-\xi, t-\tau) d \xi
$$

and consider each integral separately using the convention of constants. We write $A$ as a sum of integrals $A=A_{1}+A_{2}+A_{3}$ and treat each integral separately as follows:

$$
\begin{aligned}
A_{1} & =\int_{0}^{1} \sum_{|n| \geq 2} \frac{|n|}{t-\tau} \exp \left(-\frac{n^{2}}{t-\tau}+n \frac{x-\xi}{t-\tau}\right) \gamma(x-\xi, t-\tau) d \xi \\
& \leq \frac{(4 \pi)^{-\frac{1}{2}}}{\sqrt{t-\tau}} \int_{0}^{1} \sum_{|n| \geq 2} \frac{|n|}{t-\tau} \exp \left(\frac{1-|n|}{t-\tau}\right) d \xi \\
& \leq \frac{(4 \pi)^{-\frac{1}{2}}}{\sqrt{t-\tau}} \frac{2}{t-\tau} \exp \left\{-\frac{1}{t-\tau} \sum_{n=0}^{\infty}(n+2)\left(\exp -\frac{1}{T}\right)^{n}\right\} \\
& \leq \frac{c(T)}{\sqrt{t-\tau}}
\end{aligned}
$$


On a Class of Integro-Differential Equations

165

$$
\begin{aligned}
n=1: A_{2} & =\int_{0}^{1} \frac{1}{t-\tau} \exp \left(-\frac{1}{t-\tau}+\frac{x-\xi}{t-\tau}\right) \frac{(4 \pi)^{-\frac{1}{2}}}{\sqrt{t-\tau}} \exp -\frac{(x-\xi)^{2}}{4(t-\tau)} d \xi \\
& =\frac{1}{t-\tau} \frac{(4 \pi)^{-\frac{1}{2}}}{\sqrt{t-\tau}} \int_{0}^{1} \exp -\frac{(2-(x-\xi))^{2}}{4(t-\tau)} d \xi \\
& \leq \frac{(4 \pi)^{-\frac{1}{2}}}{\sqrt{t-\tau}} \frac{1}{t-\tau} \int_{0}^{1} \exp -\frac{1}{4(t-\tau)} d \xi \\
& \leq \frac{c(T)}{\sqrt{t-\tau}}, \\
n=-1: A_{3} & =\int_{0}^{1} \frac{1}{t-\tau} \exp \left(-\frac{1}{t-\tau}-\frac{x-\xi}{t-\tau}\right) \frac{(4 \pi)^{-\frac{1}{2}}}{\sqrt{t-\tau}} \exp -\frac{(x-\xi)^{2}}{4(t-\tau)} d \xi \\
& =\frac{1}{t-\tau} \frac{(4 \pi)^{-\frac{1}{2}}}{\sqrt{t-\tau}} \int_{0}^{1} \exp -\frac{(2+(x-\xi))^{2}}{4(t-\tau)} d \xi \\
& \leq \frac{c(T)}{\sqrt{t-\tau}} .
\end{aligned}
$$

Next we turn to the integral $B=B_{1}+B_{2}+B_{3}+B_{4}$, where we look at

$$
\begin{aligned}
B_{1} & =\int_{0}^{1} \sum_{|n| \geq 2} \frac{|n-\xi|}{t-\tau} \exp \left(-\frac{n^{2}}{t-\tau}+n \frac{x+\xi}{t-\tau}-\frac{x \xi}{t-\tau}\right) \gamma(x-\xi, t-\tau) d \xi \\
& \leq \int_{0}^{1} 2 \sum_{n=2}^{\infty} \frac{n+1}{t-\tau} \exp \left(\frac{1-n}{t-\tau}\right) \frac{(4 \pi)^{-\frac{1}{2}}}{\sqrt{t-\tau}} \exp -\frac{(x-\xi)^{2}}{4(t-\tau)} d \xi \\
& \leq \int_{0}^{1} \underbrace{\frac{2(4 \pi)^{-\frac{1}{2}}}{t-\tau} \exp -\frac{1}{t-\tau} \sum_{n=0}^{\infty}(n+3)\left(\exp -\frac{1}{T}\right)^{n}}_{\leq c(T)} \frac{1}{\sqrt{t-\tau}} \underbrace{\exp -\frac{(x-\xi)^{2}}{4(t-\tau)}}_{\leq 1} d \xi \\
& \leq \frac{c(T)}{\sqrt{t-\tau}} .
\end{aligned}
$$

Then we estimate the integral $B_{2}$

$$
\begin{aligned}
n=0: \dot{B_{2}} & =\int_{0}^{1} \frac{\xi}{t-\tau} \frac{(4 \pi)^{-\frac{1}{2}}}{\sqrt{t-\tau}} \exp -\frac{(x+\xi)^{2}}{4(t-\tau)} d \xi \\
& \leq \int_{0}^{1} \frac{\xi}{t-\tau} \frac{(4 \pi)^{-\frac{1}{2}}}{\sqrt{t-\tau}} \exp -\frac{\xi^{2}}{4(t-\tau)} d \xi
\end{aligned}
$$


and substitute by $\varphi(\xi)=\xi \sqrt{4(t-\tau)}$ to gain the desired inequality

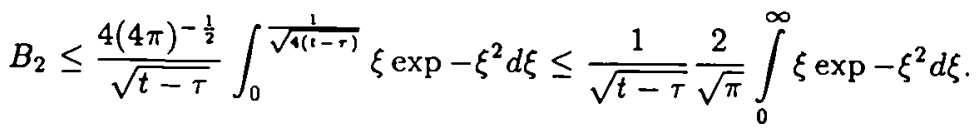

For the integral $B_{3}$ we obtain

$$
\begin{aligned}
n=1: B_{3} & =\frac{(4 \pi)^{-\frac{1}{2}}}{\sqrt{t-\tau}} \int_{0}^{1} \frac{1-\xi}{t-\tau} \exp -\frac{((1-x)+(1-\xi))^{2}}{4(t-\tau)} d \xi \\
& \leq \frac{(4 \pi)^{-\frac{1}{2}}}{\sqrt{t-\tau}} \int_{0}^{1} \frac{1-\xi}{t-\tau} \exp -\frac{(1-\xi)^{2}}{4(t-\tau)} d \xi \\
& \leq \int_{-\frac{1}{\sqrt{4(t-\tau)}}}^{0}-2 \xi \exp -\xi^{2} d \xi \frac{\pi^{-\frac{1}{2}}}{\sqrt{t-\tau}} \\
& \leq \int_{-\infty}^{0}-2 \xi \exp -\xi^{2} d \xi \frac{\pi^{-\frac{1}{2}}}{\sqrt{t-\tau}},
\end{aligned}
$$

where we applied the substitution $\psi(\xi)=1+\xi \sqrt{4(t-\tau)}$. Finally, the asserted estimation holds for the integral $B_{4}$

$$
\begin{aligned}
n=-1: B_{4} & =\int_{0}^{1} \frac{1+\xi}{t-\tau} \exp -\frac{(1+x)(1+\xi)}{t-\tau} \gamma(x-\xi, t-\tau) d \xi \\
& \leq \frac{2}{t-\tau} \exp -\frac{1}{t-\tau} \int_{0}^{1} \gamma(x-\xi, t-\tau) d \xi \\
& \leq c(T) \frac{1}{\sqrt{t-\tau}} .
\end{aligned}
$$

Considering the integral $J_{2}$ we see that the boundedness of the $\theta$-function and the substitution by $\varphi(\xi)=x+\xi \sqrt{4(t-\tau)}$ yield

$$
\begin{aligned}
J_{2} & \leq c_{1}(T) \int_{0}^{1} \frac{|x-\xi|}{2(t-\tau)} \frac{(4 \pi)^{-\frac{1}{2}}}{\sqrt{t-\tau}} \exp -\frac{(x-\xi)^{2}}{4(t-\tau)} d \xi \\
& \leq c_{1}(T) \int_{\frac{-x}{\sqrt{4(t-\tau)}}}^{\frac{1-x}{\sqrt{4(t-\tau)}}}|\xi| \exp -\xi^{2} d \xi \frac{\pi^{-\frac{1}{2}}}{\sqrt{t-\tau}} \\
& \leq c_{1}(T) \int_{-\infty}^{+\infty}|\xi| \exp -\xi^{2} d \xi \frac{\pi^{-\frac{1}{2}}}{\sqrt{t-\tau}} \\
& =c_{1}(T) \frac{\pi^{-\frac{1}{2}}}{\sqrt{t-\tau}} .
\end{aligned}
$$


Now we are able to estimate the function $q$, where

$$
q(x, t)=\iint_{0}^{t} \int_{0}^{1} \frac{\partial \Gamma}{\partial x}(x, t ; \xi, \tau) f(\xi, \tau) d \xi d \tau \quad((x, t) \in[0,1] \times[0, T])
$$

with the help of the just derived inequality as

$$
\begin{aligned}
|q(x, t)| & \leq \int_{0}^{t} \int_{0}^{1}\left|\frac{\partial \Gamma}{\partial x}(x, t ; \xi, \tau)\right||f(\xi, \tau)| d \xi d \tau \\
& \leq c(T)\|f\|_{\infty} \int_{0}^{t} \frac{1}{\sqrt{t-\tau}} d \tau \\
& =2 c(T)\|f\|_{\infty} \sqrt{t} \\
& =c_{2}(T)\|f\|_{\infty} \sqrt{t}
\end{aligned}
$$

Obviously, the function $q(\cdot, t)$ is uniformly bounded on $[0,1]$ for each $t$ and $q(x, t) \rightarrow 0$ uniformly on $[0,1]$ as $t \searrow 0$. Looking at the difference $q-\frac{\partial u_{h}}{\partial x}$, we get

$$
\left|q(x, t)-\frac{\partial u_{h}}{\partial x}(x, t)\right| \leq c_{2}(T)\|f\|_{\infty} \sqrt{h} \quad((x, t) \in[0,1] \times[\varepsilon, T]) .
$$

We conclude like in part (a) $q \in C^{0}(\bar{Q})$ with $q(x, 0)=0$ for all $x \in[0,1]$. For each $t$ the functions $u_{h}(\cdot, t)$ are continuously differentiable on $[0,1]$ and satisfy the equation $u_{h}(0, t)=0 ;$ after the fundamental theorem of calculus the identity

$$
u_{h}(x, t)=\int_{0}^{x} \frac{\partial u_{h}}{\partial x}(\xi, t) d \xi \quad(t \in[\varepsilon, T])
$$

holds, and we gain applying the uniform convergence of the functions $u_{h}$ and $\frac{\partial u_{h}}{\partial x}$ as $h \searrow 0$ on $[0,1] \times[\varepsilon, T]$ the equation

$$
u(x, t)=\int_{0}^{x} q(\xi, t) d \xi \quad((x, t) \in[0,1] \times(0, T]) .
$$

By the uniform convergence of $q(x, t)$ as $t \searrow 0$ this relationship is also true for $t=0$. Differentiating with respect to $x$ leads to $u_{x}(x, t)=q(x, t)$ on $\bar{Q}$.

Part (c): In order to show the claimed inequality, we proof first that an estimate of the type

$$
\int_{0}^{1}\left|\Gamma_{x x}(x, t ; \xi, \tau)\right| d \xi \leq c(T) \frac{1}{t-\tau} . \quad(x, \xi \in[0,1], 0 \leq \tau<t \leq T)
$$


holds. For this sake we apply the product rule and obtain

$$
\begin{aligned}
\int_{0}^{1}\left|\Gamma_{x x}(x, t ; \xi, \tau)\right| d \xi \leq & \int_{0}^{1}|\theta(x, t ; \xi, \tau)|\left|\gamma_{x x}(x-\xi, t-\tau)\right| d \xi \\
& +2 \int_{0}^{1}\left|\theta_{x}(x, t ; \xi, \tau)\right|\left|\gamma_{x}(x-\xi, t-\tau)\right| d \xi \\
& +\int_{0}^{1}\left|\theta_{x x}(x, t ; \xi, \tau)\right||\gamma(x-\xi, t-\tau)| d \xi \\
= & : J_{1}+2 J_{2}+J_{3}
\end{aligned}
$$

where each integral $J_{1}, J_{2}$ and $J_{3}$ will be investigated separately.

With regard to the integral $J_{1}$ we employ the boundedness of the $\theta$-function

$$
\begin{aligned}
J_{1} & \leq c_{1}(T) \int_{0}^{1}\left|\gamma_{x x}(x-\xi, t-\tau)\right| d \xi \\
& \leq c_{1}(T) \int_{0}^{1}\left(\frac{1}{2(t-\tau)}+\frac{(x-\xi)^{2}}{4(t-\tau)^{2}}\right) \frac{1}{\sqrt{4 \pi(t-\tau)}} \exp -\frac{(x-\xi)^{2}}{4(t-\tau)} d \xi
\end{aligned}
$$

and substitute by $\varphi(\xi)=x+\xi \sqrt{4(t-\tau)}$ to get

$$
\begin{aligned}
J_{1} & \leq c_{1}(T) \pi^{-\frac{1}{2}} \int_{\frac{-x}{\sqrt{4(t-\tau)}}}^{\frac{1-x}{\sqrt{4(t-\tau)}}}\left(\frac{1}{2(t-\tau)}+\frac{\xi^{2}}{t-\tau}\right) \exp -\xi^{2} d \xi \\
& \leq c_{1}(T) \pi^{-\frac{1}{2}} \frac{1}{(t-\tau)} \int_{-\infty}^{+\infty}\left(\frac{1}{2}+\xi^{2}\right) \exp -\xi^{2} d \xi \\
& \leq c(T) \frac{1}{t-\tau} .
\end{aligned}
$$
Next we estimate the absolute value of the integral $J_{2}$ by the sum $A+B$ of the two
integrals

$$
A=\int_{0}^{1} \sum_{n=-\infty}^{n=+\infty} \frac{|n|}{t-\tau} \exp \left(-\frac{n^{2}}{t-\tau}+n \frac{x-\xi}{t-\tau}\right) \frac{|x-\xi|}{2(t-\tau)} \frac{1}{\sqrt{4 \pi(t-\tau)}} \exp -\frac{(x-\xi)^{2}}{4(t-\tau)} d \xi
$$

and

$$
\begin{aligned}
B= & \int_{0}^{1} \sum_{n=-\infty}^{n=+\infty} \frac{|n-\xi|}{t-\tau} \exp \left(-\frac{n^{2}}{t-\tau}+n \frac{x+\xi}{t-\tau}-\frac{x \xi}{t-\tau}\right) \\
& \times \frac{|x-\xi|}{2(t-\tau)} \frac{1}{\sqrt{4 \pi(t-\tau)}} \exp -\frac{(x-\xi)^{2}}{4(t-\tau)} d \xi .
\end{aligned}
$$


Similiar estimations as in the proof of Part (b) lead to

$$
\begin{aligned}
A^{-} \leq & \frac{1}{t-\tau} \int_{0}^{1} 2 \exp \left(-\frac{1}{t-\tau}\right) \sum_{n=0}^{\infty}(n+2)\left(\exp -\frac{1}{T}\right)^{n} \frac{|x-\xi|}{2(t-\tau)} \frac{1}{\sqrt{4 \pi(t-\tau)}} d \xi \\
& +\frac{1}{t-\tau} \int_{0}^{1} \frac{|x-\xi|}{2(t-\tau)} \frac{1}{\sqrt{4 \pi(t-\tau)}} \exp -\frac{(2+(x-\xi))^{2}}{4(t-\tau)} d \xi \\
& +\frac{1}{t-\tau} \int_{0}^{1} \frac{|x-\xi|}{2(t-\tau)} \frac{1}{\sqrt{4 \pi(t-\tau)}} \exp -\frac{(2-(x-\xi))^{2}}{4(t-\tau)} d \xi \\
\leq & c(T) \frac{1}{t-\tau} .
\end{aligned}
$$

Let us write $B=B_{1}+B_{2}+B_{3}+B_{4}$. We estimate $B_{1}$ via

$$
\begin{aligned}
B_{1} \leq & \int_{0}^{1} \sum_{|n| \geq 2} \frac{|n-\xi|}{t-\tau} \exp \left(-\frac{n^{2}}{t-\tau}+n \frac{x+\xi}{t-\tau}-\frac{x \xi}{t-\tau}\right) \\
& \times \frac{|x-\xi|}{2(t-\tau)} \frac{1}{\sqrt{4 \pi(t-\tau)}} \exp -\frac{(x-\xi)^{2}}{4(t-\tau)} d \xi \\
\leq & \frac{1}{t-\tau} \int_{0}^{1} \underbrace{\sum_{n=2}^{\infty} 2(n+1) \exp \left(\frac{1-n}{t-\tau}\right) \frac{|x-\xi|}{2(t-\tau)} \frac{1}{\sqrt{4 \pi(t-\tau)}}}_{\leq c(T)} \exp -\frac{(x-\xi)^{2}}{4(t-\tau)} d \xi \\
\leq & c(T) \frac{1}{t-\tau} .
\end{aligned}
$$

Then we consider $B_{2}$ :

$$
\begin{aligned}
B_{2} & \leq \int_{0}^{1} \frac{\xi}{t-\tau} \exp \left(-\frac{x \xi}{t-\tau}\right) \frac{|x-\xi|}{2(t-\tau)} \frac{1}{\sqrt{4 \pi(t-\tau)}} \exp -\frac{(x-\xi)^{2}}{4(t-\tau)} d \xi \\
& =\frac{1}{4(\pi)^{\frac{1}{2}}} \int_{0}^{1} \frac{1}{(t-\tau)^{\frac{3}{2}}} \xi|x-\xi| \exp -\frac{(x+\xi)^{2}}{4(t-\tau)} d \xi \\
& \leq \frac{1}{4(\pi)^{\frac{1}{2}}} \frac{1}{t-\tau} \int_{0}^{1} \frac{\xi}{t-\tau} \exp \left(-\frac{(x+\xi)^{2}}{8(t-\tau)}\right) \underbrace{\frac{x+\xi}{\sqrt{t-\tau}} \exp -\frac{(x+\xi)^{2}}{8(t-\tau)}}_{\leq C} d \xi \\
& \leq C \frac{1}{t-\tau} \int_{0}^{1} \frac{\xi}{t-\tau} \exp -\frac{\xi^{2}}{8(t-\tau)} d \xi \\
& \leq c(T) \frac{1}{t-\tau} .
\end{aligned}
$$


170

W. Kohl

For the integral $B_{3}$ we obtain

$$
\begin{aligned}
B_{3} & \leq \int_{0}^{1} \frac{|1-\xi|}{t-\tau} \exp \left(-\frac{1}{t-\tau}+\frac{x+\xi}{t-\tau}-\frac{x \xi}{t-\tau}\right) \frac{|x-\xi|}{2(t-\tau)} \frac{1}{\sqrt{4 \pi(t-\tau)}} \exp -\frac{(x-\xi)^{2}}{4(t-\tau)} d \xi \\
& =\int_{0}^{1} \frac{1-\xi}{t-\tau} \exp \left(-\frac{((1-x)+(1-\xi))^{2}}{4(t-\tau)}\right) \frac{|x-\xi|}{2(t-\tau)} \frac{1}{\sqrt{4 \pi(t-\tau)}} d \xi .
\end{aligned}
$$

Using the estimation

$$
|x-\xi| \leq|x-1|+|1-\xi|=2-(x+\xi) \quad(x, \xi \in[0,1])
$$

we calculate further

$$
\begin{aligned}
B_{3} & \leq \int_{0}^{1} \frac{1-\xi}{(t-\tau)^{2}} \exp \left(-\frac{((1-x)+(1-\xi))^{2}}{8(t-\tau)}\right) \underbrace{\frac{2-(x+\xi)}{4 \sqrt{\pi} \sqrt{t-\tau}} \exp -\frac{(2-(x+\xi))^{2}}{8(t-\tau)}}_{\leq C} d \xi \\
& \leq \frac{C}{t-\tau} \int_{0}^{1} \frac{1-\xi}{t-\tau} \exp -\frac{(1-\xi)^{2}}{8(t-\tau)} d \xi \\
& \leq c(T) \frac{1}{t-\tau}
\end{aligned}
$$

At last, we proceed with the integral $B_{4}$ to get

$$
\begin{aligned}
B_{4} & \leq \int_{0}^{1} \frac{1+\xi}{t-\tau} \exp \left(-\frac{1}{t-\tau}-\frac{x+\xi}{t-\tau}-\frac{x \xi}{t-\tau}\right) \frac{|x-\xi|}{2(t-\tau)} \frac{1}{\sqrt{4 \pi(t-\tau)}} \exp -\frac{(x-\xi)^{2}}{4(t-\tau)} d \xi \\
& =\int_{0}^{1} \frac{1+\xi}{t-\tau} \exp \left(-\frac{(1+x)(1+\xi)}{t-\tau}\right) \frac{|x-\xi|}{2(t-\tau)} \frac{1}{\sqrt{4 \pi(t-\tau)}} \exp -\frac{(x-\xi)^{2}}{4(t-\tau)} d \xi \\
& \leq \int_{0}^{1} \frac{2}{t-\tau} \exp \left(-\frac{1}{t-\tau}\right) \frac{1}{2(t-\tau)} \frac{1}{\sqrt{4 \pi(t-\tau)}} d \xi \\
& \leq c(T) \frac{1}{t-\tau} .
\end{aligned}
$$

Finally, it remains to investigate the integral $J_{3}$, which we estimate by

$$
\begin{aligned}
J_{3} \leq & \int_{0}^{1} \sum_{n=-\infty}^{n=+\infty} \frac{n^{2}}{(t-\tau)^{2}} \exp \left(-\frac{n^{2}}{t-\tau}+n \frac{x-\xi}{t-\tau}\right) \frac{1}{\sqrt{4 \pi(t-\tau)}} \exp -\frac{(x-\xi)^{2}}{4(t-\tau)} d \xi \\
& +\int_{0}^{1} \sum_{n=-\infty}^{n=+\infty}\left(\frac{n-\xi}{t-\tau}\right)^{2} \exp \left(-\frac{n^{2}}{t-\tau}+n \frac{x+\xi}{t-\tau}-\frac{x \xi}{t-\tau}\right) \\
& \times \frac{1}{\sqrt{4 \pi(t-\tau)}} \exp -\frac{(x-\xi)^{2}}{4(t-\tau)} d \xi \\
= & : C+D .
\end{aligned}
$$


We treat the integral $C$ in a similiar manner as the integral $A$ above and derive without difficulties

$$
C \leq c(T) \frac{1}{t-\tau}
$$

For the integral $D_{1}$ we obtain

$$
\begin{aligned}
D_{1} \leq & \int_{0}^{1} \sum_{|n| \geq 2}\left(\frac{n-\xi}{t-\tau}\right)^{2} \exp \left(-\frac{n^{2}}{t-\tau}+n \frac{x+\xi}{t-\tau}-\frac{x \xi}{t-\tau}\right) \frac{1}{\sqrt{4 \pi(t-\tau)}} \exp -\frac{(x-\xi)^{2}}{4(t-\tau)} d \xi \\
\leq & \int_{0}^{1} \sum_{n=2}^{\infty} 2(n+1)(t-\tau)^{2} \exp \left(\frac{1-n}{t-\tau}\right) \frac{1}{\sqrt{4 \pi(t-\tau)}} \exp -\frac{(x-\xi)^{2}}{4(t-\tau)} d \xi \\
\leq & \frac{1}{t-\tau} \int_{0}^{1} \sum_{n=0}^{\infty} 2(n+3)\left(\exp -\frac{1}{T}\right)^{n} \frac{1}{t-\tau} \\
& \times \exp \left(-\frac{1}{t-\tau}\right) \frac{1}{\sqrt{4 \pi(t-\tau)}} \exp -\frac{(x-\xi)^{2}}{4(t-\tau)} d \xi \\
\leq & c(T) \frac{1}{t-\tau} .
\end{aligned}
$$

Next we go on estimating

$$
\begin{aligned}
D_{2} & =\int_{0}^{1} \frac{\xi^{2}}{(t-\tau)^{2}} \exp \left(-\frac{x \xi}{t-\tau}\right) \frac{1}{\sqrt{4 \pi(t-\tau)}} \exp -\frac{(x-\xi)^{2}}{4(t-\tau)} d \xi \\
& =\int_{0}^{1} \frac{\xi^{2}}{(t-\tau)^{2}} \frac{1}{\sqrt{4 \pi(t-\tau)}} \exp -\frac{(x+\xi)^{2}}{4(t-\tau)} d \xi \\
& \leq \frac{1}{t-\tau} \int_{0}^{1} \frac{\xi}{t-\tau} \exp \left(-\frac{\xi^{2}}{8(t-\tau)}\right) \underbrace{\frac{\xi}{\sqrt{4 \pi(t-\tau)}} \exp -\frac{\xi^{2}}{8(t-\tau)}}_{\leq C} d \xi \\
& \leq C \frac{1}{t-\tau} \int_{0}^{1} \frac{\xi}{t-\tau} \exp -\frac{\xi^{2}}{8(t-\tau)} d \xi \\
& \leq c(T) \frac{1}{t-\tau} .
\end{aligned}
$$

The integral $D_{3}$ will be estimated by

$$
\begin{aligned}
D_{3} & =\int_{0}^{1} \frac{(1-\xi)^{2}}{(t-\tau)^{2}} \exp \left(-\frac{1}{t-\tau}+\frac{x+\xi}{t-\tau}-\frac{x \xi}{t-\tau}\right) \frac{1}{\sqrt{4 \pi(t-\tau)}} \exp -\frac{(x-\xi)^{2}}{4(t-\tau)} d \xi \\
& =\int_{0}^{1} \frac{(1-\xi)^{2}}{(t-\tau)^{2}} \frac{1}{\sqrt{4 \pi(t-\tau)}} \exp -\frac{((1-x)+(1-\xi))^{2}}{4(t-\tau)} d \xi
\end{aligned}
$$




$$
\begin{aligned}
& \leq \frac{1}{t-\tau} \int_{0}^{1} \frac{1-\xi}{t-\tau} \exp \left(-\frac{(1-\xi)^{2}}{8(t-\tau)}\right) \underbrace{\frac{1-\xi}{\sqrt{4 \pi(t-\tau)}} \exp -\frac{(1-\xi)^{2}}{8(t-\tau)}}_{\leq C} d \xi \\
& \leq \frac{C}{t-\tau} \int_{0}^{1} \frac{1-\xi}{t-\tau} \exp -\frac{(1-\xi)^{2}}{8(t-\tau)} d \xi \\
& \leq c(T) \frac{1}{t-\tau} .
\end{aligned}
$$

At last we calculate for the integral $D_{4}$ without difficulties

$$
\begin{aligned}
D_{4} & =\int_{0}^{1} \frac{(1+\xi)^{2}}{(t-\tau)^{2}} \exp \left(-\frac{1}{t-\tau}-\frac{x+\xi}{t-\tau}-\frac{x \xi}{t-\tau}\right) \frac{1}{\sqrt{4 \pi(t-\tau)}} \exp -\frac{(x-\xi)^{2}}{4(t-\tau)} d \xi \\
& =\int_{0}^{1} \frac{(1+\xi)^{2}}{(t-\tau)^{2}} \exp \left(-\frac{(1+x)(1+\xi)}{t-\tau}\right) \frac{1}{\sqrt{4 \pi(t-\tau)}} \exp -\frac{(x-\xi)^{2}}{4(t-\tau)} d \xi \\
& \leq \int_{0}^{1} \frac{4}{(t-\tau)^{2}} \frac{1}{\sqrt{4 \pi(t-\tau)}} \exp -\frac{1}{t-\tau} d \xi \\
& \leq c(T) \frac{1}{t-\tau} .
\end{aligned}
$$

Now we turn to the asserted inequality of this lemma and obtain for a positive parameter $\eta$

$$
\begin{aligned}
\left|u_{x}(x+\delta, t)-u_{x}(x, t)\right| \leq & \int_{0}^{t} \int_{0}^{1} \cdot\left|\Gamma_{x}(x+\delta, t ; \xi, \tau)-\Gamma_{x}(x, t ; \xi, \tau)\right||f(\xi, \tau)| d \xi d \tau \\
\leq & \int_{0}^{t-\eta} \int_{0}^{1}\left|\Gamma_{x}(x+\delta, t ; \xi, \tau)-\Gamma_{x}(x, t ; \xi, \tau)\right||f(\xi, \tau)| d \xi d \tau \\
& +\int_{t-\eta}^{t} \int_{0}^{1}\left|\Gamma_{x}(x+\delta, t ; \xi, \tau)\right||f(\xi, \tau)| d \xi d \tau \\
& +\int_{t-\eta}^{t} \int_{0}^{1}\left|\Gamma_{x}(x, t ; \xi, \tau)\right||f(\xi, \tau)| d \xi d \tau \\
= & I_{1}+I_{2}+I_{3} .
\end{aligned}
$$

We already know by the result of part (b) that $I_{2}+I_{3} \leq 2 c_{2}(T)\|f\|_{\infty} \eta^{\frac{1}{2}}$ is true. Moreover, we obtain by the mean value theorem

$$
I_{1}=\int_{0}^{t-\eta} \int_{0}^{1}\left|\Gamma_{x x}(y, t ; \xi, \tau)\right||f(\xi, \tau)| d \xi d \tau \delta
$$


for $y$ between $x$ and $x+\delta$. We calculate further applying the inequality above

$$
\begin{aligned}
I_{1} & \leq c(T)\|f\|_{\infty} \int_{0}^{t-\eta} \frac{1}{t-\tau} d \tau \delta \\
& =c(T)\|f\|_{\infty} \delta \ln \frac{t}{\eta} \\
& \leq c(T)\|f\|_{\infty} \delta \frac{t}{\eta}
\end{aligned}
$$

Setting $\eta=\delta^{\frac{2}{3}}$ we have for all $t \in\left[\delta^{\frac{2}{3}}, T\right]$ the estimation

$$
\begin{aligned}
\left|u_{x}(x+\delta, t)-u_{x}(x, t)\right| & \leq c(T)\|f\|_{\infty} t \delta^{\frac{1}{3}}+2 c_{2}(T)\|f\|_{\infty} \delta^{\frac{1}{3}} \\
& \leq\left(c(T) T+2 c_{2}(T)\right)\|f\|_{\infty} \delta^{\frac{1}{3}} \\
& =c_{3}(T)\|f\|_{\infty} \delta^{\frac{1}{3}}
\end{aligned}
$$

whereas in the case $t \in\left[0, \delta^{\frac{2}{3}}\right]$ the inequality

$$
\begin{aligned}
\left|u_{x}(x+\delta, t)-u_{x}(x, t)\right| & \leq 2 \sup _{x \in[0,1]}\left|u_{x}(x, t)\right| \\
& \leq 2 c_{2}(T)\|f\|_{\infty} t^{\frac{1}{2}} \\
& \leq 2 c_{2}(T)\|f\|_{\infty} \delta^{\frac{1}{3}}
\end{aligned}
$$

holds

Equipped with the norm

$$
\|v\|_{C^{a, 0}(\bar{Q})}=\|v\|_{\infty}+\sup _{t \in[0, T]} h \ddot{o l} l_{\alpha}(v(\cdot, t),[0,1]),
$$

$C^{\alpha, 0}(\bar{Q})$ is a Banach space. The subspace $C_{0}^{\alpha, 0}(\bar{Q})$ consisting of all $v \in C^{\alpha, 0}(\bar{Q})$ with

$$
v(0, t)=v(1, t)=0 \quad(t \in[0, T])
$$

is a closed subspace of $C^{\alpha, 0}(\bar{Q})$, hence also a Banach space. We point out that the norm

$$
\|v\|_{C_{0}^{0}, 0(\bar{Q})}=\sup _{t \in[0, T]} h \ddot{o l} l_{\alpha}(v(\cdot, t),[0,1])
$$

is equivalent to the norm $\|\cdot\|_{C^{\alpha, 0}(\bar{Q})}$ on $C_{0}^{\alpha, 0}(\bar{Q})$. In the Banach space $C_{0}^{\alpha, 0}(\bar{Q})$ we obtain the following

Lemma 2. For $f \in C_{0}^{\alpha, 0}(\bar{Q})$, the heat potential (19) has the following properties:

(a) $u \in C^{0}(\bar{Q})$ with $\sup _{x \in[0,1]}\left|u(x, t)-\int_{0}^{t} f(x, \tau) d \tau\right| \leq c(\alpha, T)\|f\|_{C_{0}^{0.0}(\bar{Q})} t^{1+\frac{\alpha}{2}}$.

(b) $u \mid R=0$ and $\sup _{x \in[0,1]}|u(x, t)| \leq c_{1}(\alpha, T)\|f\|_{C_{0}^{\alpha, 0}(\bar{Q})} t$. 
(c) $u_{x} \in C^{0}(\bar{Q})$ with $u_{x}(x, 0) \equiv 0$ and $\sup _{x \in[0,1]}\left|u_{x}(x, t)\right| \leq c_{2}(\alpha, T)\|f\|_{C_{0}^{\alpha, 0}(\bar{Q})} t^{\frac{1+a}{2}}$.

(d) $u_{x x} \in C^{0}(\bar{Q})$ with $u_{x x} \mid R=0, u_{x x}(x, t)=\int_{0}^{t} \int_{0}^{1} \Gamma_{x x}(x, t ; \xi, \tau) f(\xi, \tau) d \xi d \tau$ and $\sup _{x \in[0,1]}\left|u_{x x}(x, t)\right| \leq c_{3}(\alpha, T)\|f\|_{C_{0}^{\alpha, 0}(\bar{Q})} t \frac{\sigma}{2}$.

(e) $u_{x x} \in C_{0}^{\alpha, 0}(\bar{Q})$, i.e. $\left|u_{x x}(x+\delta, t)-u_{x x}(x, t)\right| \leq c_{4}(\alpha, T)\|f\|_{C_{0}^{\alpha, 0}(\bar{Q})}|\delta|^{\alpha}$.

(f) $u_{t} \in C_{0}^{\alpha_{1} 0}(\bar{Q})$ with $u_{t}(x, t)=u_{x x}(x, t)+f(x, t)$ on $\bar{Q}, u_{t}(x, 0)=f(x, 0)$, and $\sup _{x \in[0,1]}\left|u_{t}(x, t)\right| \leq\|f\|_{C_{0}^{\alpha, 0}(\bar{Q})}\left(1+c_{3}(\alpha, T) t^{\frac{\alpha}{2}}\right)$.

Proof. Parts (a) and (b): By the continuity of the imbedding $C_{0}^{\alpha, 0}(\bar{Q}) \subseteq L^{\infty}(Q)$ we conclude $u \in C^{0}(\bar{Q})$ with $u \mid R=0$ and $\sup _{x \in[0,1]}|u(x, t)| \leq c_{1}(T)\|f\|_{C_{0}^{\alpha, 0}(\bar{Q})} t$. In view of the asserted inequality in statement (a) we estimate

$$
\begin{aligned}
\left|u(x, t)-\int_{0}^{\ell} f(x, \tau) d \tau\right| \leq & \int_{0}^{t}\left|\int_{0}^{1}(\Gamma(x, t ; \xi, \tau)-\gamma(x-\xi, t-\tau)) f(\xi, \tau) d \xi\right| d \tau \\
& +\left|\int_{0}^{t} \int_{0}^{1} \gamma(x-\xi, t-\tau) f(\xi, \tau) d \xi d \tau-\int_{0}^{t} f(x, \tau) d \tau\right| \\
= & : I_{1}+I_{2} .
\end{aligned}
$$

First we consider the inner integral of $I_{1}$ to obtain the estimation

$$
\left|\int_{0}^{1}(\Gamma(x, t ; \xi, \tau)-\gamma(x-\xi, t-\tau)) f(\xi, \tau) d \xi\right| \leq A+B
$$

with the integral

$$
A=\int_{0}^{1} \sum_{0 \neq n \in \mathbf{Z}} \exp \left(-\frac{n^{2}}{t-\tau}+n \frac{x-\xi}{t-\tau}\right) \gamma(x-\xi, t-\tau)|f(\xi, \tau)| d \xi
$$

and the other integral

$$
B=\int_{0}^{1} \sum_{n=-\infty}^{\infty} \exp \left(-\frac{n^{2}}{t-\tau}+n \frac{x+\xi}{t-\tau}-\frac{x \xi}{t-\tau}\right) \gamma(x-\xi, t-\tau)|f(\xi, \tau)| d \xi
$$

As usual we write $A=A_{1}+A_{2}+A_{3}$ and, obviously, we gain the inequality

$$
\begin{aligned}
A_{1} & =\int_{0}^{1} \underbrace{\sum_{|n| \geq 2} \exp \left(-\frac{n^{2}}{t-\tau}+n \frac{x-\xi}{t-\tau}\right) \frac{(t-\tau)^{-\frac{\alpha}{2}}}{\sqrt{4 \pi(t-\tau)}}}_{\leq c(T)}(t-\tau)^{\frac{\mathrm{a}}{2}} \exp -\frac{(x-\xi)^{2}}{4(t-\tau)}|f(\xi, \tau)| d \xi \\
& \leq c(T)(t-\tau)^{\frac{\alpha}{2}}\|f\|_{C_{0}^{\alpha, 0}(\bar{Q})} .
\end{aligned}
$$


For $A_{2}(n=+1)$ and $A_{3}(n=-1)$ there are no difficulties to show the same inequality, so we omit it. Considering $B=B_{1}+B_{2}+B_{3}+B_{4}$ we get for the integral $B_{1}$

$$
\begin{aligned}
B_{1}= & \int_{0}^{1} \underbrace{\sum_{|n| \geq 2} \exp \left(-\frac{n^{2}}{t-\tau}+n \frac{x+\xi}{t-\tau}-\frac{x \xi}{t-\tau}\right) \frac{(t-\tau)^{-\frac{a}{2}}}{\sqrt{4 \pi(t-\tau)}}}_{\leq c(T)} \\
& \times(t-\tau)^{\frac{0}{2}} \exp -\frac{(x-\xi)^{2}}{4(t-\tau)}|f(\xi, \tau)| d \xi \\
\leq & c(T)(t-\tau)^{\frac{a}{2}}\|f\|_{C_{0}^{a, 0}(\bar{Q})} .
\end{aligned}
$$

In the integral $B_{2}$ we apply

$$
|f(\xi, \tau)|=|f(\xi, \tau)-f(0, \tau)| \leq \xi^{\alpha}\|f\|_{C_{0}^{\alpha, 0}(\bar{Q})}
$$

and derive the inequality

$$
\begin{aligned}
B_{2} & \leq \int_{0}^{1} \frac{\xi^{\alpha}}{\sqrt{4 \pi(t-\tau)}} \exp -\frac{x \xi}{t-\tau} \exp -\frac{(x-\xi)^{2}}{4(t-\tau)} d \xi\|f\|_{C_{0}^{\alpha, 0}(\bar{Q})} \\
& =\int_{0}^{1} \frac{\xi^{\alpha}}{\sqrt{4 \pi(t-\tau)}} \exp -\frac{(x+\xi)^{2}}{4(t-\tau)} d \xi\|f\|_{C_{0}^{\alpha, 0}(\bar{Q})^{\circ}}
\end{aligned}
$$

Then we estimate further

$$
B_{2} \leq \int_{0}^{1} \frac{\xi^{\alpha}}{\sqrt{4 \pi(t-\tau)}} \exp -\frac{\xi^{2}}{4(t-\tau)} d \xi\|f\|_{C_{0}^{\alpha, 0}(\bar{Q})}
$$

and substitute by $\varphi(\xi)=\xi \sqrt{4(t-\tau)}$ to obtain

$$
\begin{aligned}
B_{2} & \leq \int_{-\infty}^{+\infty} \xi^{\alpha} \exp -\xi^{2} d \xi(4(t-\tau))^{\frac{\alpha}{2}} 4 \pi^{-\frac{1}{2}}\|f\|_{C_{0}^{\alpha, 0}(\bar{Q})} \\
& \leq c(\alpha, T)(t-\tau)^{\frac{\alpha}{2}}\|f\|_{C_{0}^{\alpha, 0}(\bar{Q})} .
\end{aligned}
$$

In the integral $B_{3}$ the inequality

$$
|f(\xi, \tau)|=|f(1, \tau)-f(\xi, \tau)| \leq(1-\xi)^{\alpha}|| f \|_{C_{0}^{\mathrm{a} .0}(\bar{Q})}
$$

leads us to

$$
\begin{aligned}
B_{3} & \leq \int_{0}^{1}(1-\xi)^{\alpha} \frac{1}{\sqrt{4 \pi(t-\tau)}} \exp -\frac{(1-x+1-\xi)^{2}}{4(t-\tau)} d \xi\|f\|_{C_{0}^{\alpha, 0}(\bar{Q})} \\
& \leq \int_{0}^{1}(1-\xi)^{\alpha} \frac{1}{\sqrt{4 \pi(t-\tau)}} \exp -\frac{(1-\xi)^{2}}{4(t-\tau)} d \xi\|f\|_{C_{0}^{\alpha, 0}(\bar{Q})}
\end{aligned}
$$


and substitution via $\varphi(\xi)=\xi \sqrt{4(t-\tau)}+1$ yields the inequality

$$
B_{3} \leq c(\alpha, T)(t-\tau)^{\frac{\alpha}{2}}\|f\|_{C_{0}^{\alpha, 0}(\bar{Q})} .
$$

Concerning the integral $B_{4}$ no difficulties occur in proofing the same kind of estimation. We summarize our results so far

$$
\left|\int_{0}^{1}(\Gamma(x, t ; \xi, \tau)-\gamma(x-\xi, t-\tau)) f(\xi, \tau) d \xi\right| \leq c(\alpha, T)\|f\|_{C_{0}^{\alpha, o}(\bar{Q})}(t-\tau)^{\frac{\alpha}{2}} .
$$

For the investigation of the integral $I_{2}$ we use the property $\int_{-\infty}^{+\infty} \gamma(x-\xi, t-\tau) d \xi=1$ and extend the function $f \in C_{0}^{\alpha, 0}(\bar{Q})$ by 0 in the set $\mathbb{R} \times[0, T] \backslash \bar{Q}$ to obtain the extension $\hat{f} \in C_{0}^{\alpha, 0}(\mathbb{R} \times[0, T])$ with $\|f\|_{C_{0}^{\alpha, 0}(\bar{Q})}=\|\hat{f}\|_{C_{0}^{\alpha, 0}(\mathbb{R} \times[0,1])}$,

$$
\begin{aligned}
& \left|\int_{0}^{1} \gamma(x-\xi, t-\tau) f(\xi, \tau) d \xi-\int_{-\infty}^{+\infty} \gamma(x-\xi, t-\tau) f(x, \tau) d \xi\right| \\
& \quad=\left|\int_{-\infty}^{+\infty} \gamma(x-\xi, t-\tau)(\hat{f}(\xi, \tau)-\hat{f}(x, \tau)) d \xi\right| \\
& \quad \leq \int_{-\infty}^{+\infty} \frac{1}{\sqrt{4 \pi t-\tau}}|x-\xi|^{\alpha} \exp -\frac{(x-\xi)^{2}}{4(t-\tau)} d \xi\|\hat{f}\|_{C_{0}^{\alpha, 0}(\mathbb{R} \times[0,1])}
\end{aligned}
$$

Finally, substitution with $\varphi(\xi)=x+\xi \sqrt{4(t-\tau)}$ yields the inequality

$$
\left|\int_{0}^{1} \gamma(x-\xi, t-\tau) f(\xi, \tau) d \xi-f(x, \tau)\right| \leq c(\alpha, T)\|f\|_{C_{0}^{a, 0}(\bar{Q})}(t-\tau)^{\frac{\alpha}{2}} .
$$

Therefore we deduce

$$
I_{1}+I_{2} \leq c(\alpha, T)\|f\|_{C_{0}^{\mathrm{a}, 0}(\bar{Q})} t^{1+\frac{\alpha}{2}}
$$

and our assertion is proved.

Part (c): Obviously, we may apply Lemma 1 to get $u_{x} \in C^{0}(\bar{Q})$ and $u_{x}(x, 0)=0$ for all $x \in[0,1]$. In order to proof the inequality

$$
\left|u_{x}(x, t)\right| \leq c_{2}(\alpha, T)\|f\|_{C_{0}^{a, 0}(\bar{Q})} t^{\frac{1+\alpha}{2}}
$$

it suffices to convince ourselves that both inequalities

$$
\begin{aligned}
I_{1}: & =\int_{0}^{1}\left|\theta_{x}(x, t ; \xi, \tau) \gamma(x-\xi, t-\tau) f(\xi, \tau)\right| d \xi \\
& \leq c(\alpha, T)\|f\|_{C_{0}^{\alpha .0}(\bar{Q})}(t-\tau)^{-\frac{1}{2}+\frac{o}{2}}
\end{aligned}
$$


and

$$
\begin{aligned}
I_{2} & :=\int_{0}^{1}\left|\theta(x, t ; \xi, \tau) \gamma_{z}(x-\xi, t-\tau) f(\xi, \tau)\right| d \xi \\
& \leq c(\alpha, T)\|f\|_{C_{0}^{\alpha .0}(\bar{Q})}(t-\tau)^{-\frac{1}{2}+\frac{\alpha}{2}}
\end{aligned}
$$

hold. As usual we employ the integral

$$
A=\int_{0}^{1} \sum_{n=-\infty}^{n=+\infty} \frac{|n|}{t-\tau} \exp \left(-\frac{n^{2}}{t-\tau}+n \frac{x-\xi}{t-\tau}\right) \gamma(x-\xi, t-\tau) d \xi
$$

and the other integral

$$
B=\int_{0}^{1} \sum_{n=-\infty}^{n=+\infty} \frac{|n-\xi|}{t-\tau} \exp \left(-\frac{n^{2}}{t-\tau}+n \frac{x+\xi}{t-\tau}-\frac{x \xi}{t-\tau}\right) \gamma(x-\xi, t-\tau) d \xi
$$

to estimate $I_{1}$ by $I_{1} \leq A+B$. Writing $\dot{A}$ as sum of integrals $A=A_{1}+\dot{A_{2}}+A_{3}$ we get

$$
\begin{aligned}
A_{1}= & \int_{0}^{1} \underbrace{\sum_{|n| \geq 2} \frac{|n|}{t-\tau} \exp \left(-\frac{n^{2}}{t-\tau}+n \frac{x-\xi}{t-\tau}\right) \frac{(4 \pi)^{-\frac{1}{2}}}{\sqrt{t-\tau}} \frac{1}{(t-\tau)^{-\frac{1}{2}+\frac{\alpha}{2}}}}_{\leq c(\alpha, T)} \\
& \times(t-\tau)^{-\frac{1}{2}+\frac{\alpha}{2}} \exp -\frac{(x-\xi)^{2}}{4(t-\tau)}|f(\xi, \tau)| d \xi \\
\leq & c(\alpha, T)\|f\|_{C_{0}^{\alpha, o}(\bar{Q})}(t-\tau)^{-\frac{1}{2}+\frac{\alpha}{2}}
\end{aligned}
$$

and remark that we can reach the same estimation for the integrals $A_{2}$ and $A_{3}$.

In view of $B=B_{1}+B_{2}+B_{3}+B_{4}$ we obtain for $B_{1}$ the estimation

$$
\begin{aligned}
B_{1} \leq & \int_{0}^{1} \sum_{|n| \geq 2} \frac{|n-\xi|}{t-\tau} \exp \left(-\frac{n^{2}}{t-\tau}+n \frac{x+\xi}{t-\tau}-\frac{x \xi}{t-\tau}\right) \frac{(4 \pi)^{-\frac{1}{2}}}{(t-\tau)^{\frac{\alpha}{2}}} \\
& \times(t-\tau)^{-\frac{1}{2}+\frac{a}{2}} \exp -\frac{(x-\xi)^{2}}{4(t-\tau)}|f(\xi, \tau)| d \xi \\
\leq & c(\alpha, T)\|f\|_{C_{0}^{a .0}(\bar{Q})}(t-\tau)^{-\frac{1}{2}+\frac{\alpha}{2}} .
\end{aligned}
$$

The integral $B_{2}$ is treated by

$$
\begin{gathered}
\int_{0}^{1} \frac{\xi}{t-\tau} \frac{(4 \pi)^{-\frac{1}{2}}}{\sqrt{t-\tau}} \exp -\frac{x \xi}{t-\tau} \exp -\frac{(x-\xi)^{2}}{4(t-\tau)}|f(\xi, \tau)| d \xi \\
\leq \int_{0}^{1} \frac{\xi^{1+\alpha}}{t-\tau} \frac{(4 \pi)^{-\frac{1}{2}}}{\sqrt{t-\tau}} \exp -\frac{(x+\xi)^{2}}{4(t-\tau)} d \xi\|f\|_{C_{0}^{\alpha, 0}(\bar{Q})} \\
\leq \int_{0}^{1} \frac{\xi^{1+\alpha}}{t-\tau} \frac{(4 \pi)^{-\frac{1}{2}}}{\sqrt{t-\tau}} \exp -\frac{\xi^{2}}{4(t-\tau)} d \xi\|f\|_{C_{0}^{\alpha, 0}(\bar{Q})}
\end{gathered}
$$


and substitution with $\varphi(\xi)=\xi \sqrt{4(t-\tau)}$ leads to

$$
B_{2} \leq c(\alpha, T)\|f\|_{C_{0}^{\alpha, 0}(\bar{Q})}(t-\tau)^{-\frac{1}{2}+\frac{\alpha}{2}}
$$

The integral $B_{3}$ will be estimated in the following way:

$$
\begin{aligned}
B_{3} & \leq \int_{0}^{1} \frac{1-\xi}{t-\tau} \frac{(4 \pi)^{-\frac{1}{2}}}{\sqrt{t-\tau}}(1-\xi)^{\alpha} \exp -\frac{((1-x)+(1-\xi))^{2}}{4(t-\tau)} d \xi\|f\|_{C_{0}^{\alpha, 0}(\bar{Q})} \\
& \leq \int_{0}^{1} \frac{(1-\xi)^{1+\alpha}}{t-\tau} \frac{(4 \pi)^{-\frac{1}{2}}}{\sqrt{t-\tau}} \exp -\frac{(1-\xi)^{2}}{4(t-\tau)} d \xi\|f\|_{C_{0}^{\alpha, 0}(\bar{Q})}
\end{aligned}
$$

Now substitution with $\varphi(\xi)=\xi \sqrt{4(t-\tau)}+1$ yields the desired estimation

$$
B_{3} \leq c(\alpha, T)\|f\|_{C_{0}^{\alpha, 0}(\bar{Q})}(t-\tau)^{-\frac{1}{2}+\frac{\alpha}{2}}
$$

For the integral $B_{4}(n=-1)$ we obtain without difficulties the same kind of estimation. In order to reach the desired inequality for the integral $I_{2}$ we estimate it with the help of two integrals

$$
\begin{aligned}
I_{2} \leq & \int_{0}^{1}|\theta(x, t ; \xi, \tau)-1|\left|\gamma_{x}(x-\xi, t-\tau)\right||f(\xi, \tau)| d \xi \\
& +\left|\int_{0}^{1} \gamma_{x}(x-\xi, t-\tau) f(\xi, \tau) d \xi\right| \\
= & : J_{1}+J_{2} .
\end{aligned}
$$

We estimate $J_{1} \leq C+D$ with the integrals

$$
C=\int_{0}^{1} \sum_{n=-\infty}^{n=+\infty} \exp \left(-\frac{n^{2}}{t-\tau}+n \frac{x-\xi}{t-\tau}\right)\left|\gamma_{x}(x-\xi, t-\tau)\right| d \xi
$$

and

$$
D=\int_{0}^{1} \sum_{n=-\infty}^{n=+\infty} \exp \left(-\frac{n^{2}}{t-\tau}+n \frac{x+\xi}{t-\tau}-\frac{x \xi}{t-\tau}\right)\left|\gamma_{x}(x-\xi, t-\tau)\right| d \xi
$$

The integral $C$ can be treated in the usual way, so we turn at once to the integral $D=D_{1}+D_{2}+D_{3}+D_{4}$. Here we restrict ourselves to the investigation of the integrals $D_{2}$ and $D_{3}$, because the way to estimate the other two integrals is clear. For the integral 
$D_{2}$ we obtain

$$
\begin{gathered}
\int_{0}^{1} \frac{|x-\xi|}{2(t-\tau)} \frac{(4 \pi)^{-\frac{1}{2}}}{\sqrt{t-\tau}} \exp -\frac{x \xi}{t-\tau} \exp -\frac{(x-\xi)^{2}}{4(t-\tau)}|f(\xi, \tau)| d \xi \\
\leq \int_{0}^{1} \xi^{\alpha} \frac{|x+\xi|}{2(t-\tau)} \frac{(4 \pi)^{-\frac{1}{2}}}{\sqrt{t-\tau}} \exp -\frac{(x+\xi)^{2}}{4(t-\tau)} d \xi\|f\|_{C_{0}^{\alpha, 0}(\bar{Q})} \\
\leq \int_{0}^{1} \frac{(x+\xi)^{1+\alpha}}{2(t-\tau)} \frac{(4 \pi)^{-\frac{1}{2}}}{\sqrt{t-\tau}} \exp -\frac{(x+\xi)^{2}}{4(t-\tau)} d \xi\|f\|_{C_{0}^{\alpha, 0}(\bar{Q})}
\end{gathered}
$$

and substitution via $\varphi(\bar{\xi})=\xi \sqrt{4(t-\tau)}-x$ leads to the inequality

$$
D_{2} \leq c(\alpha, T)\|f\|_{C_{0}^{\alpha, 0}(\bar{Q})}(t-\tau)^{-\frac{1}{2}+\frac{\alpha}{2}} .
$$

At last we estimate the integral $D_{3}$ in the following way:

$$
\begin{gathered}
\int_{0}^{1} \frac{|x-\xi|}{2(t-\tau)} \frac{(4 \pi)^{-\frac{1}{2}}}{\sqrt{t-\tau}} \exp \left(-\frac{1}{t-\tau}+\frac{x+\xi}{t-\tau}-\frac{x \xi}{t-\tau}\right) \exp -\frac{(x-\xi)^{2}}{4(t-\tau)}|f(\xi, \tau)| d \xi \\
\leq \int_{0}^{1}(1-\xi)^{\alpha} \frac{2-(x+\xi)}{2(t-\tau)} \frac{(4 \pi)^{-\frac{1}{2}}}{\sqrt{t-\tau}} \exp -\frac{(2-(x+\xi))^{2}}{4(t-\tau)} d \xi\|f\|_{C_{0}^{a, o}(\bar{Q})} \\
\leq \int_{0}^{1} \frac{(2-(x+\xi))^{1+\alpha}}{2(t-\tau)} \frac{(4 \pi)^{-\frac{1}{2}}}{\sqrt{t-\tau}} \exp -\frac{(2-(x+\xi))^{2}}{4(t-\tau)} d \xi\|f\|_{C_{0}^{\alpha, 0}(\bar{Q})} .
\end{gathered}
$$

Then we substitute by $\varphi(\xi)=\xi \sqrt{4(t-\tau)}-x+2$ to derive the desired inequality.

With regard to the integral $J_{2}$ we apply the identity $\int_{-\infty}^{+\infty} \gamma_{x}(x-\xi, t-\tau) d \xi=0$ and employ the extended function $\hat{f} \in C_{0}^{\alpha, 0}(\mathbb{R} \times[0, T])$ of the function $f$ (see p. 176) to get

$$
\begin{aligned}
J_{2} & =\left|\int_{0}^{1} \gamma_{x}(x-\xi, t-\tau) f(\xi, \tau) d \xi-\int_{-\infty}^{+\infty} \gamma_{x}(x-\xi, t-\tau) d \xi f(x, \tau)\right| \\
& \leq \int_{-\infty}^{+\infty} \gamma_{x}(x-\xi, t-\tau)|\hat{f}(\xi, \tau)-\hat{f}(x, \tau)| d \xi \\
& \leq \int_{-\infty}^{+\infty} \frac{|x-\xi|^{1+\alpha}}{2(t-\tau)} \frac{(4 \pi)^{-\frac{1}{2}}}{\sqrt{t-\tau}} \exp -\frac{(x-\xi)^{2}}{4(t-\tau)} d \xi\|f\|_{C_{0}^{\alpha, 0}(\bar{Q})} .
\end{aligned}
$$

Finally the substitution $\varphi(\xi)=\xi \sqrt{4(t-\tau)}+x$ yield inequality $(26)$. 
Part (d): To derive the existence of the second derivative $u_{x x}$ of the heat potential we show that the inequality

$$
\left|\int_{0}^{1} \Gamma_{x x}(x, t ; \xi, \tau) f(\xi, \tau) d \xi\right| \leq c(\alpha, T)\|f\|_{C_{0}^{\alpha, 0}(\bar{Q})}(t-\tau)^{\frac{\alpha}{2}-1}
$$

holds. After applying the product rule we estimate this integral by four integrals:

$$
\begin{aligned}
& \left|\int_{0}^{1} \Gamma_{x x}(x, t ; \xi, \tau) f(\xi, \tau) d \xi\right| \\
& \leq \int_{0}^{1}\left|\theta_{x x}(x, t ; \xi, \tau) \gamma(x-\xi, t-\tau) f(\xi, \tau)\right| d \xi \\
& +2 \int_{0}^{1}\left|\theta_{x}(x, t ; \xi, \tau) \gamma_{x}(x-\xi, t-\tau) f(\xi, \tau)\right| d \xi \\
& +\int_{0}^{1}\left|(\theta(x, t ; \xi, \tau)-1) \gamma_{x x}(x-\xi, t-\tau) f(\xi, \tau)\right| d \xi \\
& +\left|\int_{0}^{1} \gamma_{x x}(x-\xi, t-\tau) f(\xi, \tau) d \xi\right| \\
& =: I_{1}+2 I_{2}+I_{3}+I_{4} \text {. }
\end{aligned}
$$

These integrals will be investigated separately. First we estimate the integral $I_{1}$ by the sum of the two integrals

$$
A=\int_{0}^{1} \sum_{n=-\infty}^{n=+\infty} \frac{n^{2}}{(t-\tau)^{2}} \exp \left(-\frac{n^{2}}{t-\tau}+n \frac{x-\xi}{t-\tau}\right) \frac{1}{\sqrt{4 \pi(t-\tau)}} \exp -\frac{(x-\xi)^{2}}{4(t-\tau)}|f(\xi, \tau)| d \xi
$$

and

$$
\begin{aligned}
B= & \int_{0}^{1} \sum_{n=-\infty}^{n=+\infty}\left(\frac{|n-\xi|}{t-\tau}\right)^{2} \exp \left(-\frac{n^{2}}{t-\tau}+n \frac{x+\xi}{t-\tau}-\frac{x \xi}{t-\tau}\right) \\
& \times \frac{1}{\sqrt{4 \pi(t-\tau)}} \exp -\frac{(x-\xi)^{2}}{4(t-\tau)}|f(\xi, \tau)| d \xi
\end{aligned}
$$

We turn at once to the two interesting parts of the integral $B$. For the one part we 
obtain

$$
\begin{gathered}
\int_{0}^{1} \frac{\xi^{2}}{(t-\tau)^{2}} \exp \left(-\frac{x \xi}{t-\tau}\right) \frac{1}{\sqrt{4 \pi(t-\tau)}} \exp -\frac{(x-\xi)^{2}}{4(t-\tau)}|f(\xi, \tau)| d \xi \\
\leq \int_{0}^{1} \frac{\xi^{2+\alpha}}{(t-\tau)^{2}} \frac{1}{\sqrt{4 \pi(t-\tau)}} \exp -\frac{\xi^{2}}{4(t-\tau)} d \xi\|f\|_{C_{0}^{\alpha, 0}(\bar{Q})} \\
\leq c(\alpha, T)\|f\|_{C_{0}^{\alpha, 0}(\bar{Q})}(t-\tau)^{\frac{\alpha}{2}-1}
\end{gathered}
$$

where we employed the substitution $\varphi(\xi)=\xi \sqrt{4(t-\tau)}$. Similiar calculations lead to

$$
\begin{aligned}
& \int_{0}^{1} \frac{(1-\xi)^{2}}{(t-\tau)^{2}} \exp \left(-\frac{1}{t-\tau}+\frac{x+\xi}{t-\tau}-\frac{x \xi}{t-\tau}\right) \frac{1}{\sqrt{4 \pi(t-\tau)}} \exp -\frac{(x-\xi)^{2}}{4(t-\tau)}|f(\xi, \tau)| d \xi \\
& \quad \leq \int_{0}^{1} \frac{(1-\xi)^{2+\alpha}}{(t-\tau)^{2}} \frac{1}{\sqrt{4 \pi(t-\tau)}} \exp -\frac{(1-\xi)^{2}}{4(t-\tau)} d \xi\|f\|_{C_{0}^{\alpha, 0}(\bar{Q})} \\
& \quad \leq \int_{0}^{1} \frac{(1-\xi)^{2+\alpha}}{(t-\tau)^{2}} \frac{1}{\sqrt{4 \pi(t-\tau)}} \exp -\frac{(1-\xi)^{2}}{4(t-\tau)} d \xi\|f\|_{C_{0}^{\text {o, }}(\bar{Q})} \\
& \leq c(\alpha, T)\|f\|_{C_{0}^{\alpha, 0}(\bar{Q})}(t-\tau)^{\frac{\alpha}{2}-1}
\end{aligned}
$$

for the other part. In the treatment of the integral $I_{2}$ we proceed in the same way. We have

$$
\begin{aligned}
& \int_{0}^{1} \frac{\xi}{t-\tau} \exp \left(-\frac{x \xi}{t-\tau}\right) \frac{|x-\xi|}{2(t-\tau)} \frac{1}{\sqrt{4 \pi(t-\tau)}} \exp -\frac{(x-\xi)^{2}}{4(t-\tau)}|f(\xi, \tau)| d \xi \\
& \leq \int_{0}^{1} \frac{(x+\xi)^{2+\alpha}}{2(t-\tau)^{2}} \frac{1}{\sqrt{4 \pi(t-\tau)}} \exp -\frac{(x+\xi)^{2}}{4(t-\tau)} d \xi\|f\|_{C_{0}^{\alpha, 0}(\bar{Q})} \\
& \leq c(\alpha, T)\|f\|_{C_{0}^{\alpha, 0}(\bar{Q})}(t-\tau)^{\frac{\alpha}{2}-1}
\end{aligned}
$$

where $c(\alpha, t)$ is a positive constant obtained via the transformation $\varphi(\xi)=\xi \sqrt{4(t-T)}-$ $x$. Then we estimate

$$
\begin{aligned}
& \int_{0}^{1} \frac{1-\xi}{t-\tau} \exp \left(-\frac{1}{t-\tau}+\frac{x+\xi}{t-\tau}-\frac{x \xi}{t-\tau}\right) \frac{1}{\sqrt{4 \pi(t-\tau)}} \frac{|x-\xi|}{2(t-\tau)} \exp -\frac{(x-\xi)^{2}}{4(t-\tau)}|f(\xi, \tau)| d \xi \\
& \quad \leq \int_{0}^{1} \frac{(1-\xi)^{1+\alpha}}{t-\tau} \frac{1}{\sqrt{4 \pi(t-\tau)}} \frac{2-x-\xi}{2(t-\tau)} \exp -\frac{(2-x-\xi)^{2}}{4(t-\tau)} d \xi\|f\|_{C_{0}^{\alpha, 0}(\bar{Q})} \\
& \quad \leq \int_{0}^{1} \frac{(2-x-\xi)^{2+\alpha}}{t-\tau} \frac{1}{\sqrt{4 \pi(t-\tau)}} \exp -\frac{(2-x-\xi)^{2}}{4(t-\tau)} d \xi\|f\|_{C_{0}^{\alpha .0}(\bar{Q})} \\
& \quad \leq c(\alpha, T)\|f\|_{C_{0}^{a .0}(\bar{Q})}(t-\tau)^{\frac{\alpha}{2}-1}
\end{aligned}
$$


using the substitution $\varphi(\xi)=\xi \sqrt{4(t-T)}+x-2$. Also, by the integral $I_{3}$ we restrict ourselves to the following two cases. First we calculate

$$
\begin{aligned}
& \int_{0}^{1} \exp \left(-\frac{x \xi}{t-\tau}\right) \frac{1}{\sqrt{4 \pi(t-\tau)}}\left(\frac{1}{2(t-\tau)}+\frac{(x-\xi)^{2}}{4(t-\tau)^{2}}\right) \exp -\frac{(x-\xi)^{2}}{4(t-\tau)}|f(\xi, \tau)| d \xi \\
& \quad \leq \int_{0}^{1} \frac{1}{\sqrt{4 \pi(t-\tau)}} \xi^{\alpha}\left(\frac{1}{2(t-\tau)}+\frac{(x-\xi)^{2}}{4(t-\tau)^{2}}\right) \exp -\frac{(x+\xi)^{2}}{4(t-\tau)}|f(\xi, \tau)| d \xi \\
& \quad \leq \int_{0}^{1} \frac{1}{\sqrt{4 \pi(t-\tau)}}\left(\frac{(x+\xi)^{\alpha}}{2(t-\tau)}+\frac{(x+\xi)^{2+\alpha}}{4(t-\tau)^{2}}\right) \exp -\frac{(x+\xi)^{2}}{4(t-\tau)} d \xi\|f\|_{C_{0}^{a, 0}(\bar{Q})} \\
& \quad \leq c(\alpha, T)\|f\|_{C_{0}^{\circ, 0}(\bar{Q})}(t-\tau)^{\frac{\alpha}{2}-1}
\end{aligned}
$$

where we used the substitution $\varphi(\xi)=\xi \sqrt{4(t-\tau)}-x$. Next we estimate

$$
\begin{aligned}
& \int_{0}^{1} \exp \left(-\frac{1}{t-\tau}+\frac{x+\xi}{t-\tau}-\frac{x \xi}{t-\tau}-\frac{(x-\xi)^{2}}{4(t-\tau)}\right) \\
& \times \frac{(4 \pi)^{-\frac{1}{2}}}{\sqrt{(t-\tau)}}\left(\frac{1}{2(t-\tau)}+\frac{(x-\xi)^{2}}{4(t-\tau)^{2}}\right)|f(\xi, \tau)| d \xi \\
& \leq \int_{0}^{1} \frac{1}{\sqrt{4 \pi(t-\tau)}}\left(\frac{(x-\xi)^{\alpha}}{2(t-\tau)}+\frac{(x-\xi)^{2+\alpha}}{4(t-\tau)^{2}}\right) \exp -\frac{(2-x-\xi)^{2}}{4(t-\tau)} d \xi\|f\|_{C_{0}^{\alpha, 0}(\bar{Q})} \\
& \leq \int_{0}^{1} \frac{1}{\sqrt{4 \pi(t-\tau)}}\left(\frac{(2-x-\xi)^{\alpha}}{2(t-\tau)}+\frac{(2-x-\xi)^{2+\alpha}}{4(t-\tau)^{2}}\right) \exp -\frac{(2-x-\xi)^{2}}{4(t-\tau)} d \xi\|f\|_{C_{0}^{\alpha, 0}(\bar{Q})} \\
& \leq c(\alpha, T)\|f\|_{C_{0}^{\alpha, 0}(\bar{Q})}(t-\tau)^{\frac{\alpha}{2}-1}
\end{aligned}
$$

employing the substitution $\varphi(\xi)=\xi \sqrt{4(t-\tau)}+2-x$.

At last, it remains to look at the integral $I_{4}$. Here we apply the identity $\int_{-\infty}^{+\infty} \gamma_{x x}(x-$ $\xi, t-\tau) d \xi=0$ and use the extension $\hat{f}$ of the function $f$ (see p. 176) to get

$$
\begin{aligned}
I_{4} & =\left|\int_{0}^{1} \gamma_{x x}(x-\xi, t-\tau) f(\xi, \tau) d \xi\right| \\
& =\left|\int_{-\infty}^{+\infty} \gamma_{x x}(x-\xi, t-\tau)(\hat{f}(\xi, \tau)-\hat{f}(x, \tau)) d \xi\right| \\
& \leq \int_{-\infty}^{+\infty}\left|\gamma_{x x}(x-\xi, t-\tau)\right||\hat{f}(\xi, \tau)-\hat{f}(x, \tau)| d \xi
\end{aligned}
$$




$$
\leq \int_{-\infty}^{+\infty} \frac{1}{\sqrt{4 \pi(t-\tau)}}\left(\frac{|x-\xi|^{\alpha}}{2(t-\tau)}+\frac{(x-\xi)^{\alpha+2}}{4(t-\tau)^{2}}\right) \exp -\frac{(x-\xi)^{2}}{4(t-\tau)} d \xi\|f\|_{C_{0}^{\alpha, 0}(\bar{Q})} .
$$

Substituting with $\varphi(\xi)=x+\xi \sqrt{4(t-\tau)}$ yields the asserted inequality. Now we may conclude that the function

$$
p(x, t)=\int_{0}^{t} \int_{0}^{1} \Gamma_{x x}(x, t, \xi, \tau) f(\xi, \tau) d \xi d \tau
$$

is well-defined and satisfies the inequality

$$
\begin{aligned}
|p(x, t)| & \leq \int_{0}^{t}\left|\int_{0}^{1} \Gamma_{x x}(x, t, \xi, \tau) f(\xi, \tau) d \xi\right| d \tau \\
& \leq c(\alpha, T)\|f\|_{C_{0}^{\alpha, 0}(\bar{Q})} \int_{0}^{t} \frac{1}{(t-\tau)^{\frac{\alpha}{2}-1}} d \tau \\
& =c(\alpha, T)\|f\|_{C_{0}^{\alpha, 0}(\bar{Q})} t^{\frac{\alpha}{2}} .
\end{aligned}
$$

Hence the function $p(\cdot, t)$ is uniformly bounded on $[0,1]$ for each $t$ and $p(x, t) \rightarrow 0$ uniformly on $[0,1]$ as $t \searrow 0$. Considering the difference $p-\frac{\partial^{2} u_{h}}{\partial x^{2}}$, we obtain

$$
\left|p(x, t)-\frac{\partial^{2} u_{h}}{\partial x^{2}}(x, t)\right| \leq c(\alpha, T)\|f\|_{C_{0}^{\alpha, 0}(\bar{Q})} h^{\frac{\alpha}{2}} \quad((x, t) \in[0,1] \times[\varepsilon, T]) .
$$

We conclude similiarly as in Lemma $1 /$ Part $(\mathrm{a}), p \in C^{0}(\bar{Q})$ with $p(x, 0)=0$ for all $x \in[0,1]$. Moreover, we have $p \mid R=0$. Since the functions $\frac{\partial u_{h}}{\partial x}(\cdot, t)$ are continuously differentiable on $[0,1]$ for each $t$, we may apply the fundamental theorem of calculus to get

$$
\frac{\partial u_{h}}{\partial x}(x, t)-\frac{\partial u_{h}}{\partial x}(0, t)=\int_{0}^{x} \frac{\partial^{2} u_{h}}{\partial x^{2}}(\xi, t) d \xi \quad((x, t) \in[0,1] \times[\varepsilon, T]) .
$$

Obviously, we gain further

$$
\frac{\partial u}{\partial x}(x, t)-\frac{\partial u}{\partial x}(0, t)=\int_{0}^{x} p(\xi, t) d \xi \quad((x, t) \in[0,1] \times(0, T])
$$

as $h \searrow 0$, and this equation is also true for $t=0$. Differentiating with respect to $x$ yields $u_{x x}(x, t)=p(x, t)$ for all $(x, t) \in \bar{Q}$.

Part (e): Assuming $t \in\left[0, \delta^{2}\right]$ we calculate by virtue of Part (d)

$$
\begin{aligned}
\left|u_{x x}(x+\delta, t)-u_{x x}(x, t)\right| & \leq 2 \sup _{x \in[0,1]}\left|u_{x x}(x, t)\right| \\
& \leq 2 c_{3}(\alpha, T)\|f\|_{C_{0}^{\alpha, 0}(\bar{Q})} t^{\frac{\alpha}{2}} \\
& \leq 2 c_{3}(\alpha, T)\|f\|_{C_{0}^{\alpha, 0}(\bar{Q})} \delta^{\alpha}
\end{aligned}
$$


and the claimed inequality is valid.

In the case $t \in\left(\delta^{2}, T\right]$ we estimate with the help of three integrals

$$
\begin{aligned}
\left|u_{x x}(x+\delta, t)-u_{x x}(x, t)\right| \leq & \left|\int_{0}^{t-\delta^{2}} \int_{0}^{1}\left(\Gamma_{x x}(x+\delta, t ; \xi, \tau)-\Gamma_{x x}(x, t ; \xi, \tau)\right) f(\xi, \tau) d \xi d \tau\right| \\
& +\left|\int_{t}^{t} \int_{-\delta^{2}}^{1} \Gamma_{x x}(x+\delta, t ; \xi, \tau) f(\xi, \tau) d \xi d \tau\right| \\
& +\left|\int_{t-\delta^{2}}^{t} \int_{0}^{1} \Gamma_{x x}(x, t ; \xi, \tau) f(\xi, \tau) d \xi d \tau\right| \\
= & I_{1}+I_{2}+I_{3} .
\end{aligned}
$$

Of course, we deduce a suitable inequality for $I_{2}+I_{3}$

$$
I_{2}+I_{3} \leq 2 c(\alpha, T)\|f\|_{C_{0}^{a, 0}(\bar{Q})}\left(\delta^{2}\right)^{\frac{\alpha}{2}} .
$$

So it remains to consider the integral $I_{1}$. Applying the mean value theorem we obtain for the inner integral of $I_{1}$

$$
\int_{0}^{1} \Gamma_{x x x}(y, t ; \xi, \tau) f(\xi, \tau) d \xi \delta
$$

where $y$ lies between $x$ and $x+\delta$. The product rule and further estimations lead to the investigation of integrals which have the form

$$
A_{k l}=\int_{0}^{1} \mid \frac{\partial^{k} \dot{\theta}}{\partial x^{k}}(y, t ; \xi, \tau) \frac{\partial^{l} \gamma}{\partial x^{l}}(y-\xi, t-\tau) f\left(\xi, \tau \mid d \xi \quad\left(k+l=3, k, l \in N_{0}\right) .\right.
$$

We remark that each integral may be estimated by

$$
A_{k l} \leq c(\alpha, T)\|f\|_{C_{0}^{a \cdot 0}(\bar{Q})}(t-\tau)^{-\frac{3}{2}+\frac{a}{2}}
$$

using simliar calculations as in Part (d). Hence we know

$$
\begin{aligned}
I_{1} & \leq c(\alpha, T)\|f\|_{C_{0}^{\alpha, 0}(\bar{Q})} \int_{0}^{t-\delta^{2}}(t-\tau)^{-\frac{3}{2}+\frac{\alpha}{2}} d \tau \delta \\
& =c(\alpha, T)\|f\|_{C_{0}^{\alpha, 0}(\bar{Q})}\left(\left.\frac{2}{1-\alpha}(t-\tau)^{\frac{-1+\alpha}{2}}\right|_{0} ^{t-\delta^{2}}\right) \delta \\
& =c(\alpha, T)\|f\|_{C_{0}^{\alpha, 0}(\bar{Q})} \frac{2}{1-\alpha}\left(\delta^{-1+\alpha}-t^{\frac{-1+\alpha}{2}}\right) \delta \\
& \leq c(\alpha, T) \frac{2}{1-\alpha}\|f\|_{C_{0}^{\alpha, 0}(\bar{Q})} \delta^{\alpha}
\end{aligned}
$$


and our assertion is proved.

Part (f): We conclude with the help of the results of Part (a) and the inequalities

$$
\begin{aligned}
& \left|\frac{u(x, t)-u(x, 0)}{t}-f(x, 0)\right| \\
& \quad \leq\left|\frac{u(x, t)}{t}-\frac{1}{t} \int_{0}^{t} f(x, \tau) d \tau\right|+\left|\frac{1}{t} \int_{0}^{t}(f(x, \tau)-f(x, 0)) d \tau\right| \\
& \quad \leq c(\alpha, T)\|f\|_{C_{0}^{a, 0}(\bar{Q})} t^{\frac{\alpha}{2}}+\frac{1}{t} \int_{0}^{t}|f(x, \tau)-f(x, 0)| d \tau \\
& \quad \rightarrow 0
\end{aligned}
$$

as $t \searrow 0$, uniformly for all $x \in[0,1]$, and this yields the property $u_{t}(x, 0)=f(x, 0)$.

Next we consider the estimation

$$
\begin{aligned}
& \left|\int_{0}^{1} \Gamma(x, t ; \xi, t-h) f(\xi, t-h) d \xi-f(x, t)\right| \\
& \leq\left|\int_{0}^{1}(\Gamma(x, t ; \xi, t-h)-\gamma(x-\xi, h)) f(\xi, t-h) d \xi\right| \\
& \quad+\left|\int_{0}^{1} \gamma(x-\xi, h) f(\xi, t-h) d \xi-f(x, t-h)\right| \\
& \quad+|f(x, t-h)-f(x, t)| \\
& =: I_{1}+I_{2}+I_{3} .
\end{aligned}
$$

Applying inequalities (23) and (24) for $\tau=t-h$ we get

$$
I_{1}+I_{2} \leq 2 c(\alpha, T)\|f\|_{C_{0}^{\alpha, 0}(\bar{Q})} h^{\frac{\alpha}{2}},
$$

and from the uniform continuity of the function $f$ on $[0,1] \times[\varepsilon, T]$ we deduce the relationship

$$
\int_{0}^{1} \Gamma(x, t ; \xi, t-h) f(\xi, t-h) d \xi \rightarrow f(x, t) \quad \text { as } h \searrow 0 \text { uniformly on }[0,1] \times[\varepsilon, T] .
$$

With regard to equality (18) we notice that

$$
\frac{\partial u_{h}}{\partial t}(x, t) \rightarrow \frac{\partial^{2} u}{\partial x^{2}}(x, t)+f(x, t) \quad \text { as } h \searrow 0 \text { uniformly on }[0,1] \times[\varepsilon, T] .
$$

Hence $u_{t}(x, t)$ exists for all $(x, t) \in[0,1] \times(0, T]$. We include the case $t=0$ to yield

$$
u_{t}(x, t)=u_{x x}(x, t)+f(x, t) \quad((x, t) \in \bar{Q}) .
$$

The properties $u_{t} \in C_{0}^{\alpha, 0}(\bar{Q})$ and $\sup _{x \in[0,1]}\left|u_{t}(x, t)\right| \leq\|f\|_{C_{0}^{a, 0}(\bar{Q})}\left(1+c_{3}(\alpha, T) t^{\frac{\alpha}{2}}\right)$ follow now from the identity above in connection with Part (d) 


\section{The Barbashin operator}

In this section we state sufficient conditions under which both (4) and (5) are continuous operator functions mapping $C_{0}^{\alpha, 0}(\bar{Q})$ into itself and estimate their norm.

Lemma 3. Suppose that $c \in C^{\alpha, 0}(\bar{Q})$. Then the corresponding multiplication operator (4) is bounded in $C_{0}^{\alpha, 0}(\bar{Q})$ and $\|C\| \leq\|c\|_{C^{a, 0}(\bar{Q})}$.

Proof. From the definition of $C_{0}^{\alpha, 0}(\bar{Q})$ and the hypothesis on the function $c$ we conclude directly that $C u \in C^{0}(\bar{Q})$ for $u \in C_{0}^{\alpha, 0}(\bar{Q})$ and that the function $C u$ satisfies the boundary condition (22). From the estimates

$$
\begin{aligned}
\mid c(x, t) & u(x, t)-c(y, t) u(y, t) \mid \\
& \leq|c(x, t) u(x, t)-c(x, t) u(y, t)|+|c(x, t) u(y, t)-c(y, t) u(y, t)| \\
& \leq|c(x, t)||u(x, t)-u(y, t)|+|c(x, t)-c(y, t)||u(y, t)| \\
& \leq\|c\|_{\infty} h \ddot{o} l_{\alpha}(u(\cdot, t),[0,1])|x-y|^{\alpha}+h o l_{\alpha}(c(\cdot, t),[0,1])|x-y|^{\alpha}\|u\|_{\infty} \\
& \leq h \ddot{o} l_{\alpha}(u(\cdot, t),[0,1])|x-y|^{\alpha}|| c \|_{C^{\alpha, 0}(\bar{Q})}
\end{aligned}
$$

it follows that $C u \in C_{0}^{\alpha, 0}(\bar{Q})$ and $\|C\| \leq\|c\|_{C^{\alpha, 0}(\bar{Q})}$ erties:

Lemma 4. Suppose that the function $k:[0,1] \times[0,1] \rightarrow \mathbb{R}$ has the following prop-

(a) $k(\cdot, x)$ is measurable for each $x \in[0,1]$.

(b) $k(s, \cdot) \in C^{\alpha}([0,1])$ uniformly for all $s \in[0,1]$, i.e. there exists a constant $q \in \mathbb{R}$ with

$$
|k(s, x)-k(s, y)| \leq \tilde{q}|x-y|^{\alpha} \quad(s \in[0,1]) .
$$

(c) $k(s, 0)=k(s, 1)=0$ for all $s \in[0,1]$.

Then the corresponding partial integral operator $(5)$ is bounded in $C_{0}^{\alpha, 0}(\bar{Q})$ with $\|K\| \leq$ $\frac{q}{\alpha+1}$, where

$$
q=\sup _{s \in[0,1]} h \ddot{l} l_{\alpha}(k(s, \cdot),[0,1]) .
$$

Proof. The function $F(\cdot, x, t)=k(\cdot, x) u(\cdot, t)$ is measurable and bounded on the interval $[0,1]$ for fixed $(x, t) \in[0,1] \times[0, T]$, while the function $F(s, \cdot, \cdot)=k(s, \cdot) u(s, \cdot)$ is continuous on $\bar{Q}$ for fixed $s \in[0,1]$. Since

$$
|F(s, x, t)| \leq q\|u\|_{\infty} \quad((s, x, t) \in[0,1] \times[0,1] \times[0, T])
$$

we conclude that the integral over $F(\cdot, x, t)$ depends continuously on the parameters $x \in[0,1]$ and $t \in[0, T]$; this means that $K^{-} u \in C^{0}(\bar{Q})$. It is clear that the function $K u$ 
fulfills the boundary condition (22). Finally, from

$$
\begin{aligned}
|K u(x, t)-K u(y, t)| & =\left|\int_{0}^{1}[k(s, x)-k(s, y)] u(s, t) d s\right| \\
& \leq \int_{0}^{1}|k(s, x)-k(s, y)||u(s, t)-u(0, t)| d s \\
& \leq \sup _{s \in[0,1]} h \ddot{o l} l_{\alpha}(k(s, \cdot),[0,1])|x-y|^{\alpha} \int_{0}^{1}|u(s, t)-u(0, t)| d s \\
& \leq q|x-y|^{\alpha} \int_{0}^{1} s^{\alpha} h \ddot{o} l_{\alpha}(u(\cdot, t),[0,1]) d s
\end{aligned}
$$

we get

$$
\frac{|K u(x, t)-K u(y, t)|}{|x-y|^{\alpha}} \leq q h \ddot{o} l_{\alpha}(u(\cdot, t),[0,1]) \int_{0}^{1} s^{\alpha} d s
$$

hence

$$
h \ddot{o l} l_{\alpha}(K u(\cdot, t),[0,1]) \leq \frac{q}{\alpha+1} h \ddot{l_{\alpha}}(\dot{u}(\cdot, t),[0,1])
$$

Passing to the supremum in the interval $[0, T]$ leads to $\|K u\|_{C_{0}^{\alpha, 0}(\bar{Q})} \leq \frac{q}{\alpha+1}\|u\|_{C_{0}^{\alpha, 0}(\bar{Q})}$ as claimed

\section{The linear problem}

Now we turn from the parabolic differential equation (6) to the equivalent operator equation (8). We calculate the spectral radius of the operator $L^{-1}(C+K)$ and give existence and uniqueness results for equation (6)

First of all, we need the following

Lemma 5. For $f \in C_{0}^{\alpha, 0}(\bar{Q})$, the following two statements are equivalent:

(A) $u \in C^{0}(\bar{Q})$ has the properties $u_{x} \in C^{0}(\bar{Q}), u_{1}, u_{x x} \in C^{0}(Q)$ and solves the boundary value problem

$$
\left.\begin{array}{rlrl}
L u & =(C+K) u+f & & \text { in } Q \\
u & =0 & & \text { on } R .
\end{array}\right\}
$$

(B) $u \in C_{0}^{\alpha, 0}(\bar{Q})$ satisfies the linear operator equation (8).

Proof. Let $u$ be as in statement (A). We fix $(x, t) \in Q$ and observe that for $0<$ $t_{0}<t$ the vector field $F:[0,1] \times\left[0, t_{0}\right] \rightarrow \mathbb{R}^{2}$ defined by

$$
F(\xi, \tau)=\left(\Gamma(x, t ; \xi, \tau) u_{\xi}(\xi, \tau)-\Gamma_{\xi}(x, t ; \xi, \tau) u(\xi, \tau),-\Gamma(x, t ; \xi, \tau) u(\xi, \tau)\right)
$$


is continuous on $[0,1] \times\left[0, t_{0}\right]$ and continuously differentiable on $(0,1) \times\left(0, t_{0}\right)$ with

$$
\begin{aligned}
\operatorname{div} F(\xi, \tau) & =-\Gamma(x, t ; \xi, \tau) L u(\xi, \tau)-u(\xi, \tau)\left[\Gamma_{\xi \xi}(x, t ; \xi, \tau)+\Gamma_{r}(x, t ; \xi, \tau)\right] \\
& =-\Gamma(x, t ; \xi, \tau)[(C+K) u(\xi, \tau)+f(\xi, \tau)] .
\end{aligned}
$$

So the divergence of the vector field $F$ is continuous and bounded on $(0,1) \times\left(0, t_{0}\right)$ and we may apply the Gauss theorem to obtain

$$
\int_{0}^{t_{0}} \int_{0}^{1}-\Gamma(x, t ; \xi, \tau)[(C+K) u(\xi, \tau)+f(\xi, \tau)] d \xi d \tau=\int_{0}^{1}-\Gamma\left(x, t ; \xi, t_{0}\right) u\left(\xi, t_{0}\right) d \xi
$$

Letting $t_{0} \rightarrow t$ we get the identity

$$
\int_{0}^{t} \int_{0}^{1} \Gamma(x, t ; \xi, \tau)[(C+K) u(\xi, \tau)+f(\xi, \tau)] d \xi d \tau=u(x, t) \quad((x, t) \in Q) .
$$

The function on the left-hand side of (31) is continuous on $\bar{Q}$ by Lemma 1 and we have $u \in C^{0}(\bar{Q})$, so the above equation holds for all $(x, t) \in \bar{Q}$. Of course, $u \in C_{0}^{\alpha, 0}(\bar{Q})$ and $\left[I-L^{-1}(C+K)\right] u=L^{-1} f$.

Conversely, let $u$ be as in (B). Since $f \in C_{0}^{\alpha, 0}(\bar{Q})$, the same is true for the function $(C+K) u+f$. Moreover, from the identity $L^{-1}[(C+K) u+f]=u$ and from Lemmas 1 and 2 it follows that the function $u$ has the regularity properties stated in (A) and satisfies (30)

Lemma 6. The spectral radius $r(A)$ of the operator $A=L^{-1}(C+K): C_{0}^{\alpha, 0}(\bar{Q}) \rightarrow$ $C_{0}^{\alpha, 0}(\bar{Q})$ is zero.

Proof. We use the classical Gel'fand formula

$$
r(A)=\lim _{n \rightarrow \infty} \sqrt[n]{\left\|A^{n}\right\|}
$$

First of all, the inequalities $\|C v\|_{\infty} \leq\|c\|_{\infty}\|v\|_{\infty}$ and $\|K v\|_{\infty} \leq q\|v\|_{\infty}$, with $q$ as in (29), combined with property (a) in Lemma 1 , lead to the estimate

$$
|A v(x, t)| \leq t c_{1}\left(\|c\|_{\infty}+q\right)\|v\|_{\infty} .
$$

By induction, we get then

$$
\left|A^{n} v(x, t)\right| \leq \frac{t^{n}}{n !}\left[c_{1}\left(\|c\|_{\infty}+q\right)\right]^{n}\|v\|_{\infty} \quad(n \in \mathbb{N})
$$

Furthermore, for arbitrary $x, z \in[0,1]$ we have, by the mean value theorem,

$$
\begin{aligned}
& \frac{\left|A^{n} v(x, t)-A^{n} v(z, t)\right|}{|x-z|^{\alpha}} \\
& \leq \frac{\left|A^{n} v(x, t)-A^{n} v(z, t)\right|}{|x-z|} \\
& \quad=\left|\int_{0}^{t} \int_{0}^{1} \Gamma_{x}(y, t ; \xi, \tau)(C+K) A^{n-1} v(\xi, \tau) d \xi d \tau\right| \\
& =: I(y)
\end{aligned}
$$


for some $y$ between $x$ and $z$. Applying inequalities (20) and (32) we obtain

$$
\begin{aligned}
I(y) & \leq \int_{0}^{t} \int_{0}^{1}\left|\Gamma_{x}(y, t ; \xi, \tau)\right|\left[|c(\xi, \tau)|\left|A^{n-1} v(\xi, \tau)\right|+q \int_{0}^{1}\left|A^{n-1} v(s, \tau)\right| d s\right] d \xi d \tau \\
& \leq \int_{0}^{t} \frac{c_{2}}{\sqrt{t-\tau}}\left(\|c\|_{\infty}+q\right) \frac{\tau^{n-1}}{(n-1) !}\left[c_{1}\left(\|c\|_{\infty}+q\right)\right]^{n-1}\|v\|_{\infty} d \tau \\
& \leq\left(\int_{0}^{t} \frac{\tau^{n-1}}{\sqrt{t-\tau}} \frac{1}{(n-1) !} d \tau\right)\left[a\left(\|c\|_{\infty}+q\right)\right]^{n}\|v\|_{\infty}
\end{aligned}
$$

with $a=\max \left\{c_{1}, c_{2}\right\}$. The identity

$$
\int_{0}^{t} \frac{\tau^{n-1}}{\sqrt{t-\tau}} \frac{1}{(n-1) !} d \tau=\frac{2^{n}}{1 \cdot 3 \cdot 5 \cdots(2 n-1)} t^{n-\frac{1}{2}}
$$

leads to

$$
\begin{aligned}
& \left(\int_{0}^{t} \frac{\tau^{n-1}}{\sqrt{t-\tau}} \frac{1}{(n-1) !} d \tau\right)\left[a\left(\|c\|_{\infty}+q\right)\right]^{n}\|v\|_{\infty} \\
& \leq \frac{2^{n}}{1 \cdot 3 \cdot 5 \cdots(2 n-1)} t^{n-\frac{1}{2}}\left[a\left(\|c\|_{\infty}+q\right)\right]^{n}\|v\|_{\infty} \\
& \leq \frac{\left[2 a T\left(\|c\|_{\infty}+q\right)\right]^{n}}{n !} T^{-\frac{1}{2}}\|v\|_{\infty}
\end{aligned}
$$

Consequently, we obtain the estimate

$$
\left\|A^{n} v\right\|_{C_{0}^{a, 0}(\bar{Q})} \leq \frac{\left[2 a T\left(\|c\|_{\infty}+q\right)\right]^{n}}{n !} T^{-\frac{1}{2}}\|v\|_{C_{0}^{a, 0}(\bar{Q})} \quad(n \in \mathbb{N}) .
$$

From this estimate we deduce

as claimed

$$
\sqrt[n]{\left\|A^{n}\right\|} \leq 2 a T\left(\|c\|_{\infty}+q\right) \sqrt[n]{T^{-\frac{1}{2}}} \sqrt[n]{\frac{1}{n !}} \rightarrow 0 \quad(n \rightarrow \infty)
$$

Building on the results of the previous sections we are now able to prove the following

Theorem 1. The inhomogeneous linear equation (8) has for each $f \in C_{0}^{\alpha, 0}(\bar{Q})$ a unique solution $u \in C_{0}^{\alpha, 0}(\bar{Q})$. This solution can be represented as infinite series

$$
u=\sum_{n=0}^{\infty}\left[L^{-1}(C+K)\right]^{n}\left(L^{-1} f\right)
$$

and depends continuously on the data $f \in C_{0}^{\alpha, 0}(\bar{Q})$.

Proof. The operator $A=L^{-1}(C+K)$ is a continuous endomorphism of the Banach space $C_{0}^{\alpha, 0}(\bar{Q})$. From Lemma 6 we know that the Neumann series $\sum_{n=0}^{\infty} A^{n}$ converges to the inverse of the operator $I-A$. Consequently, for $f \in C_{0}^{\alpha, 0}(\bar{Q})$ the inhomogeneous linear equation (8) has a unique solution $u=(I-A)^{-1}\left(L^{-1} f\right) \in C_{0}^{\alpha, 0}(\bar{Q})$ which depends continuously on $f$ and has the representation (34) 

by

From the proof of Lemma 6 we see that the norm of $(I-A)^{-1}$ may be estimated

$$
\left\|(I-A)^{-1}\right\| \leq 1+\sum_{n=1}^{\infty}\left\|A^{n}\right\| \leq 1+\frac{\exp \left[2 a T\left(\|c\|_{\infty}+q\right)\right]-1}{\sqrt{T}} .
$$

Next we consider the Dirichlet problem for the linear equation (8) with prescribed boundary function $\varphi$, which belongs to the set

$$
C^{1}(R)=\left\{\varphi \in C^{0}(R) \mid \varphi(\cdot, 0) \in C^{1}([0,1]) \text { and } \varphi(0, \cdot), \varphi(1, \cdot) \in C^{1}([0, T])\right\} .
$$

Theorem 2. Let $f \in C_{0}^{\alpha, 0}(\bar{Q})$ and $\varphi \in C^{1}(R)$. Then the problem

$$
\left.\begin{array}{rlrl}
L u & =(C+K) u+f & & \text { in } Q \\
u(x, 0) & =\varphi(x, 0) & & (x \in[0,1]) \\
u(0, t) & =\varphi(0, t) & & (t \in(0, T)) \\
u(1, t) & =\varphi(1, t) & & (t \in(0, T])
\end{array}\right\}
$$

has a unique solution $u \in C^{0}(\bar{Q})$ with $u_{x} \in C^{0}(\bar{Q})$ and $u_{t}, u_{x x} \in C^{0}(Q)$.

Proof. If $u_{1}$ and $u_{2}$ are two solutions of problem (35), we see that the function $u=u_{1}-u_{2}$ solves problem (30), and hence $u \equiv 0$

As usual, we obtain a representation of a solution $u$ of problem (35) if we add the solution of problem (30) to the solution of the homogeneous heat equation $L u=0$ with $u \mid R=\varphi$, which we denote by $S \varphi$, with

$$
\begin{aligned}
S \varphi(x, t)= & \int_{0}^{1} \Gamma(x, t ; \xi, 0) \varphi(\xi, 0) d \xi \\
& +\int_{0}^{t} \Gamma_{\xi}(x, t ; 0, \tau) \varphi(0, \tau) d \tau-\int_{0}^{t} \Gamma_{\xi}(x, t ; 1, \tau) \varphi(1, \tau) d \tau .
\end{aligned}
$$

So we have explicitly

$$
u(x, t)=\sum_{n=0}^{\infty}\left[L^{-1}(C+K)\right]^{n}\left(L^{-1} f\right)(x, t)+S \varphi(x, t) .
$$




\section{The nonlinear problem}

In the nonlinear case we first give sufficient conditions under which the nonlinear operator $K H$, with $K$ given by (5) and $H$ given by (10), acts on $C_{0}^{\alpha, 0}(\bar{Q})$ and satisfies a Lipschitz condition in order to apply a classical fixed point principle.

Lemma 7. Suppose that the function $k:[0,1] \times[0,1] \rightarrow \mathbb{R}$ satisfies the three conditions (a) - (c) stated in Lemma 4. Moreover, let $h: \bar{Q} \times \mathbb{R} \rightarrow \mathbb{R}$ be a continuous function satisfying a Lipschitz condition

$$
|h(x, t, u)-h(x, t, v)| \leq L|u-v| .
$$

Then the nonlinear operator $K H$ acts on $C_{0}^{\alpha, 0}(\bar{Q})$ with

$$
\left.\begin{array}{c}
\|K H u-K H v\|_{\infty} \leq q L\|u-v\|_{\infty} \\
\|K H u-K H v\|_{C_{0}^{\alpha .0}(\bar{Q})} \leq q L\|u-v\|_{C_{0}^{a} \cdot(\bar{Q})}
\end{array}\right\}
$$

where $q$ is given by (29).

Proof. It is easy to see that the function $K H u$ is continuous if $u$ is continuous. Furthermore, the function $K H u$ satisfies the boundary condition $K H u(0, t)=K H u(1, t)=$ 0 for all $t \in[0, T]$. From the estimate

$$
\begin{aligned}
|K H u(x, t)-K H u(y, t)| & \leq \int_{0}^{1}|k(s, x)-k(s, y)||h(s, t, u(s, t))| d s \\
& \leq q|x-y|^{\alpha} \max _{(s, t) \in \bar{Q}}|h(s, t, u(s, t))|
\end{aligned}
$$

we see that the operator $K H$ maps $C^{0}(\bar{Q})$ into $C_{0}^{\alpha, 0}(\bar{Q})$, and hence $K H: C_{0}^{\alpha, 0}(\bar{Q}) \rightarrow$ $C_{0}^{\alpha, 0}(\bar{Q})$. For functions $u, v \in C_{0}^{\alpha, 0}(\bar{Q})$ we have .

$$
\begin{aligned}
& \frac{|(K H u-K H v)(x, t)-(K H u-K H v)(y, t)|}{|x-y|^{\alpha}} \\
& \quad \leq \frac{1}{|x-y|^{\alpha}} \int_{0}^{1}|k(s, x)-k(s, y)||h(s, t, u(s, t))-h(s, t, v(s, t))| d s \\
& \quad \leq q L\|u-v\|_{\infty} \\
& \quad \leq q L\|u-v\|_{C_{0}^{\alpha, 0}(\bar{Q})} .
\end{aligned}
$$

From this the assertion follows

In view of the nonlinear operator equation (12) with imposed boundary conditions we define the function spaces

$$
C_{0}^{1}([0,1])=\left\{g \in C^{1}([0,1]) \mid g(0)=g(1)=0\right\}
$$


and

$$
C_{0}^{1,0}(\bar{Q})=\left\{u \mid u, u_{x} \in C^{0}(\bar{Q}) \text { and } u(0, t)=u(1, t)=0 \text { for all } t \in[0, T]\right\} .
$$

Equipped with the norms

$$
\|g\|_{C_{0}^{1}([0,1])}=\sup _{x \in[0,1]}\left|g^{\prime}(x)\right| \quad \text { and } \quad\|u\|_{C_{0}^{1,0}(\bar{Q})}=\sup _{(x, t) \in \bar{Q}}\left|u_{x}(x, t)\right|,
$$

respectively, both function spaces are Banach spaces, and we can state the following

Lemma 8. For $g \in C_{0}^{1}([0,1])$, the boundary operator $S$ with

$$
(S g)(x, t)=\int_{0}^{1} \Gamma(x, t ; \xi, 0) g(\xi) d \xi
$$

is a continuous operator from $C_{0}^{1}([0,1])$ into $C_{0}^{1,0}(\bar{Q})$ and $\|S\|=1$.

Proof. The function $r=S g$ satisfies the homogenuous heat equation $\operatorname{Lr}(x, t)=0$ for all $(x, t) \in Q$ with the boundary conditions $r(x, 0)=g(x)$ for all $x \in[0,1]$ and $r(0, t)=r(1, t)=0$ for all $t \in[0, T]$. If we extend $g$ to the odd function $\tilde{g}$ on the interval $[-1,1]$ and continue $\tilde{g}$ to the periodic function $\hat{g}$ with period 2 , we remark that the function $\int_{-\infty}^{+\infty} \gamma(x-\xi, t) \hat{g}(\xi) d \xi$ is also a solution of the Dirichlet problem above. Hence a unicity argument yields

$$
r(x, t)=\int_{-\infty}^{+\infty} \gamma(x-\xi, t) \hat{g}(\xi) d \xi \quad((x, t) \in \bar{Q}) .
$$

Obviously, $\hat{g}$ and $\hat{g}^{\prime}$ are bounded and continuous functions on the whole real line. First we have $r \in C^{0}(\bar{Q})$. Considering the difference quotients

$$
\begin{aligned}
I_{h} & =\frac{r(x+h, t)-r(x, t)}{h} \\
& =\frac{1}{h}\left(\int_{-\infty}^{+\infty} \gamma(x+h-\xi, t) \hat{g}(\xi) d \xi-\int_{-\infty}^{+\infty} \gamma(x-\xi, t) \hat{g}(\xi) d \xi\right) \\
& =\int_{-\infty}^{+\infty} \gamma(\xi, t) \frac{\hat{g}(\xi+x+h)-\hat{g}(\xi+x)}{h} d \xi
\end{aligned}
$$

we notice that the integrand is dominated by

$$
\left|\gamma(\xi, t) \frac{\hat{g}(\xi+x+h)-\hat{g}(\xi+x)}{h}\right| \leq \underbrace{\gamma(\xi, t) \cdot \sup _{\mathbb{R}}\left|\hat{g}^{\prime}\right|}_{\in L^{\prime}(\mathbb{R})} \quad(\xi \in \mathbb{R}) .
$$


Now Lebesgue's Dominated Convergence Theorem insures that

$$
I_{h} \rightarrow \int_{-\infty}^{+\infty} \gamma(\xi, t) \hat{g}^{\prime}(\xi+x) d \xi=\int_{-\infty}^{+\infty} \gamma(x-\xi, t) \hat{g}^{\prime}(\xi) d \xi
$$

as $h \rightarrow 0$. Thus

$$
r_{x}(x, t)=\int_{-\infty}^{+\infty} \gamma(x-\xi, t) \hat{g}^{\prime}(\xi) d \xi \quad \text { and } \quad r_{x} \in C^{0}(\bar{Q})
$$

hold. Moreover, from the inequality

$$
\sup _{(x, t) \in \bar{Q}}\left|r_{x}(x, t)\right| \leq \int_{-\infty}^{+\infty} \gamma(x-\xi, t) d \xi \sup _{\xi \in \mathbb{R}}\left|\hat{g}^{\prime}(\xi)\right|=\sup _{\xi \in[0,1]}\left|g^{\prime}(\xi)\right|
$$

we deduce $\|S\| \leq 1$. For the function $g(x)=\sin \pi x \in C_{0}^{1}([0,1])$ we have explicitly

$$
S g(x, t)=\exp -\pi^{2} t \sin \pi x \in C_{0}^{1}(\bar{Q}) \quad \text { and } \quad\|S g\|_{C_{0}^{1}(\bar{Q})}=\|g\|_{C_{0}^{1}([0,1])}=\pi
$$

so $\|S\|$ cannot be less than 1

Before we turn to the nonlinear operator equation (12), we remark that equations (9) and (12) are equivalent. Even more is true, namely $(9) /(2)$ is equivalent to a nonlinear operator equation with an imposed boundary operator in the sense of the following.

Lemma 9. For $f \in C_{0}^{\alpha, 0}(\bar{Q})$ and $g \in C_{0}^{1}([0,1])$, the following two statements are equivalent:

(A) $u \in C^{0}(\bar{Q})$ has the properties $u_{x} \in C^{0}(\bar{Q}), u_{t}, u_{x x} \in C^{0}(Q)$ and solves the boundary value problem

$$
\left.\begin{array}{rlrl}
L u & =(C+K H) u+f & & \text { in } Q \\
u(x, 0) & =g(x) & & (x \in[0,1]) \\
u(0, t) & =u(1, t)=0 & & (t \in[0, T]) .
\end{array}\right\}
$$

(B) $u \in C_{0}^{\alpha, 0}(\bar{Q})$ satisfies the nonlinear operator equation

$$
u-L^{-1}(C+K H) u=L^{-1} f+S g .
$$

Proof. It follows the pattern of the proof of Lemma 5 with only minor modifications. Hence it is omitted 
Theorem 3. The nonlinear operator $B: C_{0}^{\alpha, 0}(\bar{Q}) \rightarrow C_{0}^{\alpha, 0}(\bar{Q})$ defined bi $B u=$ $L^{-1}(C+K H) u+L^{-1} f+S g$ has precisely one fixed point $w \in C_{0}^{\alpha, 0}(\bar{Q})$. This fixed point may be obtained as limit of the successive approximations $v_{n}=B^{n} v_{0}$ with arbitrary $v_{0} \in C_{0}^{\alpha, 0}(\bar{Q})$

Proof. First of all, the inequalities

$$
\left.\begin{array}{rl}
\|C(u-v)\| & \leq\|c\|_{\infty}\|u-v\|_{\infty} \\
\|K H u-K H v\|_{\infty} & \leq q L\|u-v\|_{\infty}
\end{array}\right\}
$$

with $q$ given by (29) and $L$ by (37), lead to

$$
\begin{aligned}
|B u(x, t)-B v(x, t)| & =\left|L^{-1} C(u-v)(x, t)+L^{-1} K(H u-H v)(x, t)\right| \\
& \leq\left|L^{-1} C(u-v)(x, t)\right|+\left|L^{-1} K(H u-H v)(x, t)\right| \\
& \leq t c_{1}\left(\|c\|_{\infty}+q L\right)\|u-v\|_{\infty} .
\end{aligned}
$$

By induction, the inequality

$$
\left|B^{n} u(x, t)-B^{n} v(x, t)\right| \leq \frac{t^{n}}{n !}\left[c_{1}\left(\|c\|_{\infty}+q L\right)\right]^{n}\|u-v\|_{\infty}
$$

may be proved for arbitrary $n \in \mathbb{N}$. In order to estimate the norm $\left\|B^{n} u-B^{n} v\right\|_{C_{0}^{a, 0}(\bar{Q})}$ we fix $x, z \in[0,1]$ and get, by the mean value theorem,

$$
\begin{aligned}
& \left|\left(B^{n} u-B^{n} v\right)(x, t)-\left(B^{n} u-B^{n} v\right)(z, t)\right| \\
& =\left|\int_{0}^{t} \int_{0}^{1}[\Gamma(x, t ; \xi, \tau)-\Gamma(z, t ; \xi, \tau)]\left[(C+K H) B^{n-1} u-(C+K H) B^{n-1} v\right](\xi, \tau) d \xi d \tau\right| \\
& =|x-z|\left|\int_{0}^{t} \int_{0}^{1} \Gamma_{x}(y ; t ; \xi, \tau)\left[(C+K H) B^{n-1} u-(C+K H) B^{n-1} v\right](\xi, \tau) d \xi d \tau\right| \\
& =: J(y)
\end{aligned}
$$

for some $y$ between $x$ and $z$. Furthermore,

$$
\begin{aligned}
J(y) & \left.\leq|x-z|^{\alpha} \int_{0}^{t} \int_{0}^{1}\left|\Gamma_{x}(y, t ; \xi, \tau)\right| \mid(C+K H) B^{n-1} u-(C+K H) B^{n-1} v\right)(\xi, \tau) \mid d \xi d \tau \\
& \leq|x-z|^{\alpha} \int_{0}^{t} \int_{0}^{1}\left|\Gamma_{x}(y, t ; \xi, \tau)\right|\left(\|c\|_{\infty}+q L\right)\left|\left(B^{n-1} u-B^{n-1} v\right)(\xi, \tau)\right| d \xi d \tau \\
& \leq|x-z|^{\alpha} \int_{0}^{t} \frac{c_{2}\left(\|c\|_{\infty}+q L\right)}{\sqrt{t-\tau}} \frac{\tau^{n-1}\left[c_{1}\left(\|c\|_{\infty}+q L\right)\right]^{n-1}\|u-v\|_{\infty}}{(n-1) !} d \tau \\
& \leq|x-z|^{\alpha}\left(\int_{0}^{t} \frac{1}{\sqrt{t-\tau}} \frac{\tau^{n-1}}{(n-1) !} d \tau\right)\left[a\left(\|c\|_{\infty}+q L\right)\right]^{n}\|u-v\|_{\infty}
\end{aligned}
$$


with $a=\max \left\{c_{1}, c_{2}\right\}$. Using again identity (33) we obtain

$$
h \ddot{o} l_{\alpha}\left(\left(B^{n} u-B^{n} v\right)(\cdot, t)\right) \leq \frac{2^{n} t^{n-\frac{1}{2}}\left[a\left(\|c\|_{\infty}+q L\right)\right]^{n}}{1 \cdot 3 \cdot 5 \cdots(2 n-1)}\|u-v\|_{\infty} .
$$

This implies that $\left\|B^{n} u-B^{n} v\right\|_{C_{0}^{\mathbf{a}, 0}(\bar{Q})} \leq d_{n}\|u-v\|_{C_{0}^{\mathrm{a}, 0}(\bar{Q})}$ where

$$
d_{n}=\frac{2^{n} T^{n-\frac{1}{2}}\left[a\left(\|c\|_{\infty}+q L\right)\right]^{n}}{1 \cdot 3 \cdot 5 \cdots(2 n-1)} .
$$

Obviously, we can choose $n_{0} \in \mathbb{N}$ such that

$$
\frac{d_{n+1}}{d_{n}^{-}}=\frac{2 T a\left(\|\dot{c}\|_{\infty}+q L\right)}{2 n+1}<\frac{3}{4}
$$

say, for $n \geq n_{0}$. Consequently, the series $\sum_{n=1}^{\infty} d_{n}$ converges. By Weissinger's fixed point theorem of [7], the operator $B$ has a unique fixed point $w \in C_{0}^{\alpha, 0}(\bar{Q})$, which can be obtained by the successive approximation $v_{n+1}={ }^{\prime} B v_{n}$, with $v_{0} \in C_{0}^{\alpha, 0}(\bar{Q})$ arbitrary. Moreover, the error estimate $\left\|w-v_{n}\right\|_{C_{0}^{\alpha, 0}(\bar{Q})} \leq\left\|B v_{0}-v_{0}\right\|_{C_{0}^{\alpha, 0}(\bar{Q})} \sum_{k=n}^{\infty} d_{k}$ is true

As a corollary of Theorem 3, we get the following

Theorem 4. Let $f \in C_{0}^{\alpha, 0}(\bar{Q})$ and $\varphi \in C^{0}(R)$. Then the problem

$$
\left.\begin{array}{rlrl}
L u & =(C+K H) u+f & & \text { in } Q \\
u & =\varphi & \text { on } R
\end{array}\right\}
$$

has a at least one solution $u \in C^{0}(\bar{Q})$ such that $u_{x}, u_{t}, u_{x x} \in C^{0}(Q)$. One solution can be represented in the form.

$$
\begin{aligned}
u(x, t)= & w(x, t)+\int_{0}^{1} \Gamma(x, t ; \xi, 0) \varphi(\xi, 0) d \xi \\
& +\int_{0}^{t} \Gamma_{\xi}(x, t ; 0, \tau) \varphi(0, \tau) d \tau-\int_{0}^{t} \Gamma_{\xi}(x, t ; 1, \tau) \varphi(1, \tau) d \tau
\end{aligned}
$$

where $w \in C_{0}^{\alpha, 0}(\bar{Q})$ is the unique fixed point of the nonlinear operator $\tilde{B} u=L^{-1}(C+$ $K H) u+L^{-1} f$.

Theorem 5. The solution of the boundary value problem (38) depends continuously on the functions $f \in C_{0}^{\alpha, 0}(\bar{Q})$ and $g \in C_{0}^{1}([0,1])$.

Proof. Given $f, h \in C_{0}^{\alpha, 0}(\bar{Q})$ and $g, j \in C_{0}^{1}([0,1])$, denote by $v, w \in C_{0}^{\alpha, 0}(\bar{Q})$ the unique solutions of the operator equations

$$
\begin{gathered}
v=L^{-1}(C+K H) v+L^{-1} f+S g \\
w=L^{-1}(C+K H) w+L^{-1} h+S j,
\end{gathered}
$$


respectively. Differencing the derivatives with respect to $x$ and estimating yields

$$
\begin{aligned}
& \left|v_{x}(x, t)-w_{x}(x, t)\right| \\
& \leq\|S g-S j\|_{C_{0}^{1,0}(\bar{Q})} \\
& \quad+\int_{0}^{t} \int_{0}^{1}\left|\Gamma_{x}(x, t ; \xi, \tau)\right|[|C v-C w|+|K H v-K H w|+|f-h|](\xi, \tau) d \xi d \tau \\
& \leq c_{2}(T) \sqrt{t}\|f-h\|_{C_{0}^{\alpha, 0}(\bar{Q})}+|| g-j \|_{C_{0}^{1}([0,1])} \\
& \quad+\int_{0}^{t} \frac{c(T)}{\sqrt{t-\tau}}\left(\|c\|_{\infty}+q L\right) \sup _{\xi \in[0,1]}|(v-w)(\xi, \tau)| d \tau .
\end{aligned}
$$

We apply the mean value theorem,

$$
|(v-w)(\xi, t)|=|(v-w)(\xi, t)-(v-w)(0, t)|=\left|\left(v_{x}-w_{x}\right)(z, t)\right||\xi|
$$

for $z \in(0, \xi)$, to obtain the relationship

$$
\sup _{\xi \in[0,1]}|(v-w)(\xi, t)| \leq \sup _{x \in[0,1]}\left|v_{x}(x, t)-w_{x}(x, t)\right|:=\varphi(t)
$$

Employing this inequality and passing to the supremum over the interval $[0,1]$ yields

$$
\varphi(t) \leq c_{2}(T) \sqrt{t}\|f-h\|_{C_{0}^{a, 0}(\bar{Q})}+\|g-j\|_{C_{0}^{1}([0,1])}+\int_{0}^{t} \frac{c(T)}{\sqrt{t-\tau}}\left(\|c\|_{\infty}+q L\right) \varphi(\tau) d \tau
$$

By virtue of the generalized Gronwall's inequality (see, e.g., [2: p. 304/ Lemma 17.7.1]) the estimate

$$
\left.\varphi(t) \leq \tilde{c}_{2}(T)\left(c_{2}(T) \sqrt{t}\|f-h\|_{C_{0}^{a, 0}(\bar{Q})}+\|g-j\|_{C_{0}^{1}([0,1])}\right)\right)
$$

holds. Hence we get the inequality

$$
\|v-w\|_{C_{0}^{1.0}(\bar{Q})} \leq \tilde{c}(T)\left(\|f-h\|_{C_{0}^{\alpha, 0}(\bar{Q})}+\|g-j\|_{C_{0}^{1}([0,1])}\right)
$$

with a certain constant $\tilde{c}(T)$. This concludes the proof

To illustrate the existence and uniqueness results of the previous section, let us consider a very simple example. Let $\omega:[0,1] \rightarrow \mathbb{R}$ be defined by

$$
\omega(x)= \begin{cases}x^{\alpha} & \text { for } 0 \leq x \leq \frac{1}{2} \\ (1-x)^{\alpha} & \text { for } \frac{1}{2} \leq x \leq 1\end{cases}
$$


Put

$$
\left.\begin{array}{rl}
k(s, x) & =\hat{k}(s) \omega(x) \\
h(x, t, u) & =\hat{h}(x, t) \arctan u \\
f(x, t) & =\omega(x) \hat{f}(t) \\
g(x) & =\sin \pi x
\end{array}\right\}
$$

where $\hat{k}:(0,1) \rightarrow \mathbb{R}$ is measureable and bounded, while $\hat{h}: Q \rightarrow \mathbb{R}$ and $\hat{f}:[0, T] \rightarrow \mathbb{R}$ are continuous. Obviously, $k$ satisfies the hypotheses of Lemma 4 with $q=\|\hat{k}\|_{L^{\infty}((0,1))}$. Moreover, $h$ satisfies (37) with $L=\max \{|\hat{h}(x, t)|:(x, t) \in \bar{Q}\}, f \in C_{0}^{\alpha, 0}(\bar{Q})$ with $\|f\|_{C_{0}^{0}, 0}(\bar{Q})=\|\hat{f}\|_{C^{0}([0, T])}$ and $g \in C_{0}^{1}([0,1])$. As multiplicator we may choose, for example, $c(x, t)=x^{\alpha} p(t)$ with $p \in C^{0}([0, T]$; from Lemma 3 we know then that $\|C\| \leq$ $2\|p\|_{C^{0}([0, T])}$. For this choice of data; the operator $C+K H$ has the form

$$
(C+K H) u(x, t)=x^{\alpha} u(x, t)+\omega(x) \int_{0}^{1} \hat{k}(s) \hat{h}(s, t) \arctan u(s, t) d s
$$

From Theorem 3 we conclude that the sequence of successive approximations

$$
\left.\begin{array}{rl}
\dot{v}_{0}(x, t) & \equiv 0 \\
& \vdots \\
v_{n+1}(x, t) & =L^{-1}(C+K H) v_{n}(x, t)+L^{-1} f(x, t)+S g(x, t)
\end{array}\right\}
$$

has a well-defined limit $w \in C_{0}^{\alpha, 0}(\bar{Q})$. If $\hat{f}(t) \equiv 0$ and $g \equiv 0$, we have of course $u(x, t) \equiv 0$, and this is the only solution of problem (38), by Lemma 9 and Theorem 3 . On the other hand, if $\hat{f}(t) \not \equiv 0$ and $g \not \equiv 0$, from Theorem 5 we may conclude not only that $u(x, t)=w(x, t)$ is the unique solution of problem $(38)$, but also that this solution depends continuously on $\hat{f}$. In particular, $u(x, t) \rightarrow 0$ uniformly on $\bar{Q}$ if $\|\hat{f}\|_{\infty} \rightarrow 0$ and $\|g\|_{C_{0}^{2}([0,1])} \rightarrow 0$.

\section{The extension of the operator $L^{-1}$}

This last section is concerned with some generalizations of the preceding results. In order to solve the inhomogenuous heat equation with zero boundary values, we chose for technical reasons the heat source $f$ from the Hölder space $\dot{C}_{0}^{\alpha, 0}(\bar{Q})$. On this space the operator $L^{-1}$ has particularly nice properties. Actually, one can take the larger Hölder space $C^{\alpha, 0}(\bar{Q})$ as underlying Banach space of the boundary value problem (7).

Together with the solution operator $S$ of the homogenuous heat equation with $C^{1}$ boundary values, where $S \varphi$ is given by (36), with the projection operator $P$,

$$
P f(x, t)=f(0, t)+x(f(1, t)-f(0, t)),
$$


and with the Volterra operator $V$,

$$
V f(x, t)=\int_{0}^{t} f(x, \tau) d \tau
$$

we can represent the unique solution of the boundary value problem (7) with the help of the extended operator $L_{c}^{-1}$

$$
L_{e}^{-1}=L^{-1}(I-P)+(I-S) V P
$$

in the form

$$
u=L_{e}^{-1} f+S \varphi .
$$

In fact, we have $u, u_{x} \in C^{0}(\bar{Q})$, and direct calculations yield $u \mid R=\varphi$ and $L u=f$.

According to the plan in the introduction we formulate now sufficient conditions that the operators $C$ and $\dot{K} H$ act continuously on $C^{\alpha, 0}(\bar{Q})$.

Lemma 10. Suppose that $c \in C^{\alpha, 0}(\bar{Q})$. Then the corresponding multiplication operator (4) is bounded in $C^{\alpha, 0}(\bar{Q})$ and $\|C\| \leq\|c\|_{C^{\alpha, 0}(\bar{Q})}$.

Lemma 11. Suppose that the function $k:[0,1] \times[0,1] \rightarrow \mathbb{R}$ has the following properties:

(a) $k(x, \cdot) \in L^{1}([0,1])$ for each $x \in[0,1]$.

(b) $k(\cdot, s) \in C^{\alpha}([0,1])$ for almost every $s \in[0,1]$, such that there exists a function $q \in L^{1}([0,1])$ with the property

$$
|k(x, \dot{s})-k(y, s)| \leq q(s)|x-y|^{\alpha} \quad \text { for a.e. } s \in[0,1] .
$$

Then the corresponding partial integral operator $(5)$ is bounded in $C^{\alpha, 0}(\bar{Q})$ with

$$
\|K\| \leq\|q\|_{L^{1}([0,1])}+\sup _{x \in[0,1]}\|k(x, \cdot)\|_{L^{1}([0,1])}
$$

Lemma 12. Suppose that the function $k:[0,1] \times[0,1] \rightarrow \mathbb{R}$ satisfies conditions (a) and (b) stated in Lemma 11. Moreover, let $h: \bar{Q} \times \mathbb{R} \rightarrow \mathbb{R}$ be a continuous function satisfying the Lipschitz condition (37). Then the nonlinear operator $K H$ acts on $C^{\alpha, 0}(\bar{Q})$ with

$$
\left.\begin{array}{c}
\|K H u-K H v\|_{\infty} \leq L \cdot \sup _{x \in[0,1]}\|k(x, \cdot)\|_{L^{1}([0,1])}\|u-v\|_{\infty} \\
\|K H u-K H v\|_{C^{\alpha}: 0(\bar{Q})} \leq L\left(\|q\|_{L^{1}([0,1])}+\sup _{x \in[0,1]}\|k(x ; \cdot)\| \|_{L^{1}([0,1])}\right)\|u-v\|_{\infty}
\end{array}\right\}
$$

We omit the proofs of these three lemmata, because their proofs follow the pattern of that given in Lemma 3, 4 and 7 with only minor modifications.

After modifying the proof of Lemma 5 we are able to establish the next result. 
Lemma 13. For $f \in C^{\alpha, 0}(\bar{Q})$ and $\varphi \in C^{1}(R)$, the following two statements are equivalent:

(A) $u \in C^{0}(\bar{Q})$ has the properties $u_{x} \in C^{0}(\bar{Q})$ and $u_{t}, u_{x x} \in C^{0}(Q)$ and solves the boundary value problem

$$
\left.\begin{array}{rlrl}
L u & =(C+K H) u+f & & \text { in } Q \\
u \mid R & =\varphi . & \text { on } R
\end{array}\right\}
$$

(B) $u \in C^{\alpha, 0}(\bar{Q})$ satisfies the nonlinear operator equation

$$
u-L_{e}^{-1}(C+K H) u=L_{e}^{-1} f+S g .
$$

Our main existence and uniqueness result reads as follows.

Theorem 6. For $f \in C^{\alpha, 0}(\bar{Q})$ and initial data $\varphi \in C^{1}(R)$ the boundary value problem

$$
\left.\begin{array}{rl}
L u & =(C+K H) u+f \\
u \mid R & =\varphi
\end{array}\right\}
$$

possesses a unique solution.

Proof. By Lemma 13 it suffices to show that the integral equation admits a unique solution. For $a<b$ the set

$$
B([a, b])=\left\{v:[0,1] \times[a, b] \rightarrow \mathbb{R} \mid v, v_{x} \in C^{0}([0,1] \times[a, b])\right\}
$$

becomes together with the norm

$$
\|v\|_{[a, b]}=\sup _{(x, t) \in[0,1] \times[a, b]}|v(x, t)|+\sup _{(x, t) \in[0,1] \times[a, b]}\left|v_{x}(x, t)\right|
$$

a Banach.space. The nonlinear operator $A u=L_{e}^{-1}(C+K H) u+L_{e}^{-1} f+S \varphi$ maps $B([0, \eta])$ into $B([0, \eta])$ for $0<\eta \leq T$. Employing Lemma 1 we know that for $v \in B([0, \eta])$ the estimation

$$
\left\|L^{-1} v\right\|_{[0, \eta]} \leq c(T)(\eta+\sqrt{\eta})\|v\|_{[0, \eta]}
$$

holds. Actually, the same kind of estimation is valid for the operator $L_{e}^{-1}$. Applying this and the assumptions on the continuity of the operator $C+K H$ we obtain the inequality

$$
\|A u-A v\|_{[0, \eta]} \leq \tilde{c}(\eta+\sqrt{\eta})\|u-v\|_{[0, \eta]}
$$

with a constant $\tilde{c}(T,\|C\|,\|K\|, L)$. Choosing $n \in \mathbb{N}$ such that the estimation

$$
\tilde{c}(\eta+\sqrt{\eta})<i
$$

holds for $\eta=\frac{T}{n}$, the operator $A$ is a contraction of $B([0, \eta])$ into $B([0, \eta])$, and according to the Banach fixed point theorem the operator $A$ possesses a unique fixed 
point $w_{1} \in B([0, \eta])$. Assuming that the integral equation possesses a unique solution $w_{k} \in B([0, k \eta])$ for $k \leq n-1$ we introduce the function $r:[0,1] \times[k \eta,(k+1) \eta] \rightarrow \mathbb{R}$,

$$
\begin{aligned}
r(x, t)= & \int_{0}^{1} \Gamma(x, t ; \xi, 0) \varphi(\xi, 0) d \xi \\
& +\int_{0}^{k \eta 1} \int_{0}^{k \eta} \Gamma(x, t ; \xi, \tau)(I-P)\left[(C+K H) w_{k}(\xi, \tau)+f(\xi, \tau)\right] d \xi d \tau \\
& +\int_{0}^{k \eta} P\left[(C+K H) w_{k}(x, \tau)+f(x, \tau)\right] d \tau \\
& -\int_{0}^{k \eta} \Gamma_{\xi}(x, t ; 0, \tau)\left(\int_{0}^{\tau}(C+K H) w_{k}(0, s)+f(0, s) d s-\varphi(0, \tau)\right) d \tau \\
& +\int_{0}^{k \eta} \Gamma_{\xi}(x, t ; 1, \tau)\left(\int_{0}^{\tau}(C+K H) w_{k}(1, s)+f(1, s) d s-\varphi(1, \tau)\right) d \tau .
\end{aligned}
$$

Next we consider the operator $A_{1}$,

$$
\begin{aligned}
A_{1} v(x, t)= & r(x, t)+\int_{k \eta 0}^{t} \int_{0}^{1} \Gamma(x, t ; \xi, \tau)(I-P)[(C+K H) v(\xi, \tau)+f(\xi, \tau)] d \xi d \tau \\
& +\int_{k \eta}^{t} P[(C+K H) v(x, \tau)+f(x, \tau)] d \tau \\
& -\int_{k \eta}^{t} \Gamma_{\xi}(x, t ; 0, \tau)\left(\int_{k \eta}^{\tau}(C+K H) v(0, s)+f(0, s) d s-\varphi(0, \tau)\right) d \tau \\
& +\int_{k \eta}^{t} \Gamma_{\xi}(x, t ; 1, \tau)\left(\int_{k \eta}^{\tau}(C+K H) v(1, s)+f(1, s) d s-\varphi(1, \tau)\right) d \tau .
\end{aligned}
$$

Of course, $A_{1}$ maps $B([k \eta,(k+1) \eta])$ into $B([k \eta,(k+1) \eta])$ and the estimate

$$
\left\|A_{1} u-A_{1} v\right\|_{[k \eta,(k+1) \eta]} \leq \tilde{c}(\eta+\sqrt{\eta})\|u-v\|_{[k \eta,(k+1) \eta]}
$$

holds. From (46), we conclude that $A_{1}$ is a contraction and hence possesses a unique fixed point $u \in B(\mid k \eta,(k+1) \eta])$. Since the fixed point of $A_{1}$ matches continuously with $w_{k}(x, k \eta)$ and $\frac{\partial}{\partial x} w_{k}(x, k \eta)$, we see that the function $w_{k+1}$,

$$
w_{k+1}(x, t)= \begin{cases}w_{k}(x, t) & \text { if }(x, t) \in[0,1] \times[0, k \eta] \\ u(x, t) & \text { if }(x, t) \in[0,1] \times[k \eta,(k+1) \eta]\end{cases}
$$

is the unique solution of the integral equation in $B([0,(k+1) \eta])$. Applying this argument we can construct inductively the unique solution of the integral equation in $B([0, T])$ 


\section{References}

[1] Appell, J., Kalitvin, A. A. and P. P. Zabrejko: Boundary value problems for integrodifferential equations of Barbashin type. J. Int. Equ. Appl. 6 (1994), 1 - 30.

[2] Cannon, J. R.: The One-Dimensional Heat Equation. Menlo Park, California: AddisonWesley 1984.

[3] Kamke, E.: Differentialgleichungen. Vol. II. Leipzig: Akad. Verlagsges. 1962.

[4] Minin, I. N: Theory of Radiation Transfer in the Atmosphere of Planets and Stars (in Russian). Moscow: Nauka 1988.

[5] Sobolev, V. V.: The Transfer of Radiation Energy in the Atmosphere of Stars and Planets (in Russian). Moscow: Gostekhizdat 1956.

[6] van der Mee, C. V. M.: Transport theory in $L_{p}$ spaces. Int. Equ. Oper. Theory 6 (1983), $405-443$.

[7] Weissinger, J.: Zur Theorie und Anwendung des Iterationsverfahrens. Math. Nachr. 8 (1952), $193-212$.

Received 13.04.1999 